\title{
Pimachrysa (Neuroptera: Chrysopidae: Nothochrysinae): Larval Description and Support for Generic Relationships
}

\author{
Catherine A. Tauber ${ }^{1,2}$ and David K. Faulkner ${ }^{3,4}$ \\ ${ }^{1}$ Department of Entomology, Comstock Hall, Cornell University, Ithaca, NY 14853-2601, USA \\ ${ }^{2}$ Department of Entomology \& Nematology, University of California, Davis, CA 95616, USA \\ ${ }^{3}$ Forensic Entomology Services, 2321 Gladwick Street, Rancho Dominguez, CA 90220, USA \\ ${ }^{4}$ Entomology Department, San Diego Natural History Museum, 1788 El Prado, San Diego, CA 92101, USA \\ Correspondence should be addressed to Catherine A. Tauber; cat6@cornell.edu
}

Received 21 June 2015; Accepted 4 October 2015

Academic Editor: G. Wilson Fernandes

Copyright (C) 2015 C. A. Tauber and D. K. Faulkner. This is an open access article distributed under the Creative Commons Attribution License, which permits unrestricted use, distribution, and reproduction in any medium, provided the original work is properly cited.

\begin{abstract}
We describe the previously unknown larval characteristics of Pimachrysa (second and third instars), and we provide new comparative data on the Nothochrysa californica Banks larvae that were described earlier. The Pimachrysa larvae (identified as Pimachrysa fusca Adams) express the numerous features that characterize the chrysopid subfamily Nothochrysinae. They resemble Hypochrysa larvae in many respects, but several differences support retaining the two genera as separate. It now appears that the reputedly more archaic, small bodied, nothochrysine genera (e.g., Dictyochrysa, Hypochrysa, and Pimachrysa) have naked larvae that differ distinctly from the larvae of Nothochrysa species, all of which are debris-carriers. In addition, the pattern of variability in Nothochrysa larval features supports the proposal that this genus has a closer relationship with the other chrysopid subfamilies than do the small-bodied nothochrysine genera. The findings are consistent with the hypothesis that larval nakedness (as opposed to debris-carrying) was the ancestral life style for the extant chrysopid clade.
\end{abstract}

\section{Introduction}

Considerable effort has focused on elucidating the evolutionary history of the neuropteran family Chrysopidae, and yet significant challenges remain. The subfamily Nothochrysinae is of particular interest, largely because of its position at or very near the base of the chrysopid phylogenetic tree and also because of its substantial and ancient fossil record. Members of this subfamily have primitive patterns of wing venation and unique features in their larval morphology that have been used to explore evolutionary trends within the subfamily [16]. However, generic relationships among the extant taxa remain ambiguous. On-going molecular studies $[4,7,8]$ thus far have yielded some interesting, but contradictory results concerning the origin of the subfamily, and they have not yet resolved the relationships among its constituent genera.

Ideally, the above issues would be met with systematic comparisons among all included taxa using a broad range of approaches (adult morphology, larval morphology, comparative biology, and molecular sequencing). In reality, a large barrier prevents such an analysis, that is, the rarity of specimens from relevant taxa. For example, except for a few species, specimens of Nothochrysinae and other basal chrysopid taxa are seldom encountered in the field; as a result the range of taxonomic coverage has been relatively narrow.

To continue efforts in overcoming this barrier, we describe the hitherto unknown larva (Semaphoront B) of the nothochrysine genus, Pimachrysa. Along with Nothochrysa, Dictyochrysa, and Hypochrysa, whose larvae were described earlier (Box 1), Pimachrysa is now the fourth genus of Nothochrysinae for which larvae are known. Also, we provide new data on the larva of Nothochrysa californica Banks that document a broader range of larval variation than previously reported for the genus. Finally, we discuss the pattern of variation among known nothochrysine larvae in relation to 
the current understanding of generic relationships and the phylogeny of the subfamily.

\section{Materials and Methods}

2.1. Larval Material and Procedures. Our study is based on four specimens of Pimachrysa fusca Adams collected in late February and late April at Lower Covington Flat, Joshua Tree National Park (previously a national monument), Riverside Co., CA. Three of the specimens (two third instars and the single second instar) were used for morphological study, and one third instar was reared to confirm the specific identity of our larval specimens. The three specimens were treated in KAAD solution [9] for 24 hours and then transferred to $95 \%$ ethyl alcohol. In the laboratory the specimens were photographed, and the external gross features were described. Subsequently, one specimen from each instar was cleared in $\mathrm{KOH}$ and transferred to glycerin for examination of fine structures and setation. The second instar died soon after it was collected. We noted a globule of apparently sticky material on the labrum and base of the left mandible; presumably the material was exudate from aphids that we supplied the larva while we were in the field. We were able to remove the exudate from the preserved specimen without damage.

All voucher specimens are now in the Tauber Research Collection (Lot 2015: 01); a leg from one of the third instars was given to Shaun Winterton, California Department of Food and Agriculture, Sacramento, for DNA analysis.

To rear one of the third instars to the adult stage, we held it in a glass vial provided with frozen eggs of Ephestia kuehniella (Zeller), the Mediterranean flour moth. It was maintained at room temperature on a long-day light: dark cycle $(\sim \mathrm{LD}$ $16: 8)$.

2.2. Terminology and Comparisons. The chrysopid larval stage typically includes three instars, the first of which (L1, Semaphoront A) differs markedly from the latter two (L2, L3), which, in turn, usually resemble each other (Semaphoront B). The main differences between second and third instars are in size, markings, and the number of thoracic and abdominal setae. In this paper, both instars of Semaphoront B are considered.

With minor exceptions, morphological terminology and chaetotaxy followed the usage of Rousset [10], Tsukaguchi [11], Tauber et al. [12], and Monserrat and Díaz-Aranda [13]. In one case there is a discrepancy. Some abdominal segments of Nothochrysa have a pair of laterodorsal tubercles (LDTs); in papers by Tauber and coauthors, the term tubercle refers to a protuberance bearing more than one seta. In Nothochrysa californica Banks, each LDT bears one long, filiform seta and one shorter seta. Thus, the tubercles of $N$. californica are consistent with those of other chrysopids. However, third instars of the two European species of Nothochrysa also were reported to have laterodorsal "tubercles" on abdominal segments A5 to A7; but in these species, the "tubercles" were reported to have only one, relatively long, seta [13]. If such a situation pertains (and no small seta is found on these "tubercles"), the homology of the structures should be examined and the usage of the term "tubercle" clarified. For convenience, in this paper, we refer to the structures reported for the European species as laterodorsal tubercles (LDTs).

For our comparisons, we used specimens of Nothochrysa californica Banks (all instars from Alameda and Marin Counties, California) and Hypochrysa africana Kimmins (second instar from South Africa, see [6]), as well as earlier descriptions in the literature, as follows: Dictyochrysa fulva EsbenPetersen (first instar) [14], Hypochrysa africana (Kimmins) (second instar) [6], Hypochrysa elegans (Burmeister) (first and third instars) [13, 15], N. californica (all instars) [16], Nothochrysa capitata (Fabricius) (third instar) [13, 17], and Nothochrysa fulviceps (Stephens) (first and third instars) [13, 17]. Our comparisons are summarized in Box 2.

\section{Background}

3.1. Habitat. The collection site (northwestern corner of Joshua Tree National Park) is in a high elevation portion of the Mohave Desert (elevation: $\sim 4670 \mathrm{ft}$ ). The area is open and arid, with scattered, low-growing trees and bushes amongst rocky outcroppings. Vegetation at the site includes Quercus cornelius-mulleri (Muller oak), Juniperus californica (California juniper), Pinus monophylla (single-leaf pinyon pine), Larrea tridentata (creosote bush), Bebbia juncea (Bebbia), Yucca brevifolia (Joshua tree), and Yucca schidigera (Mohave yucca). Our specimens were beaten from oak bushes, Q. corneliusmulleri. The collections occurred on two days: the first (a single second instar, L2) on 26-2-2015 (cool and sunny), and the second (three third instars, L3) on 28-4-2015 (warm and sunny).

3.2. Larval Behavior. The specimens did not carry debris and they gave the overall impression of a Chrysoperla larva, but their bodies appeared thicker and more rounded (Figures $1(\mathrm{a})-1(\mathrm{c}))$. They remained motionless during much of the time we observed them; occasionally they moved quickly. The third instars, in the laboratory, fed readily on eggs of $E$. kuehniella; one specimen spun a delicate, oval shaped cocoon on the side of its vial 5-6 days after captivity; small pieces of debris were incorporated into the outer layers of the cocoon. The larva (within the cocoon) remained in diapause for 4 to 5 months, pupated 155 days after spinning, and emerged as an adult 15 days later. The pharate adult exited the cocoon via a circular opening that it cut at the head end of the cocoon.

3.3. Generic Diagnosis. Our P. fusca larvae have the three diagnostic features that typify nothochrysine larvae. (i) The flagellum is very short relative to the pedicel $(\sim 1 / 14$ th the length of the pedicel) (Figure 1(d)). (ii) The terminus of the flagellum has a group of short apical setae. (iii) More than three lateral sensilla are present on the terminal segment of the labial palps (L2 = 5, L3 = 4-5) (Figure 1(e)).

Among the Nothochrysinae with known larvae, Pimachrysa (P. fusca) closely resembles Hypochrysa (two species) (Box 2). That is, the larvae (second and third instars) from both genera have elongate, naked bodies; the thorax and abdomen lack lateral tubercles; the abdomen has no laterodorsal tubercles. In addition, none of the setae are 


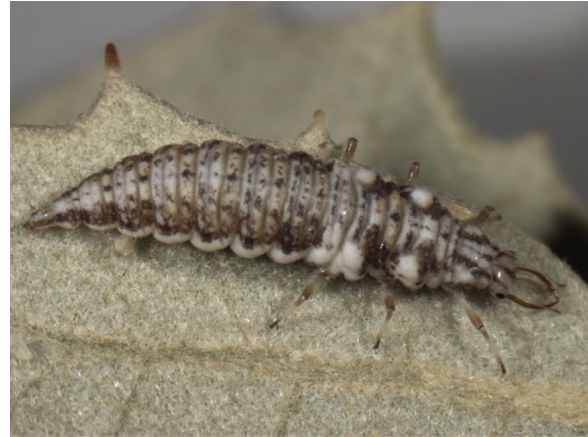

(a)

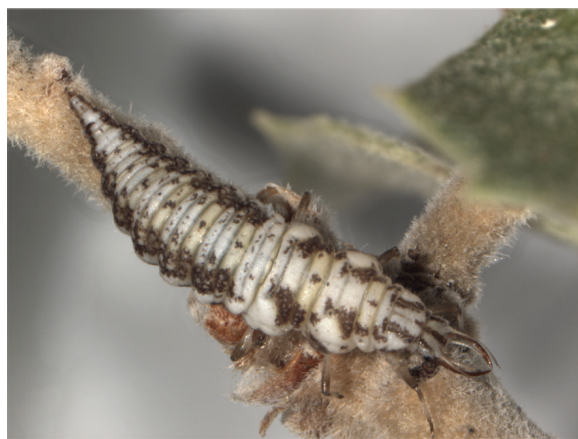

(c)

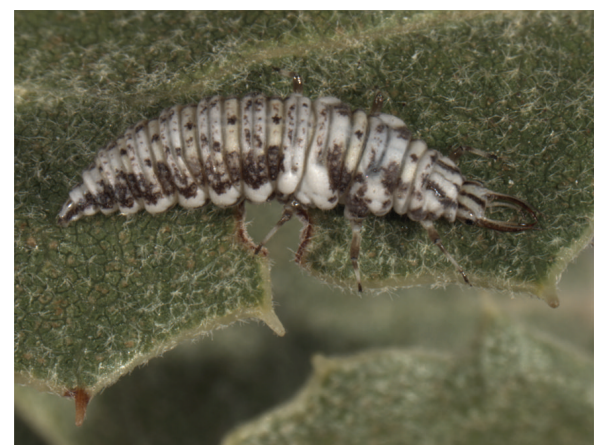

(b)

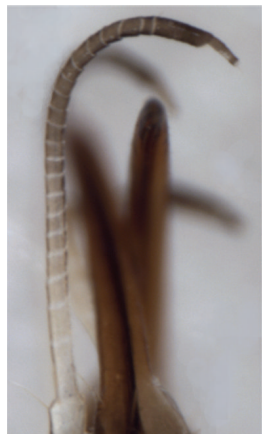

(d)

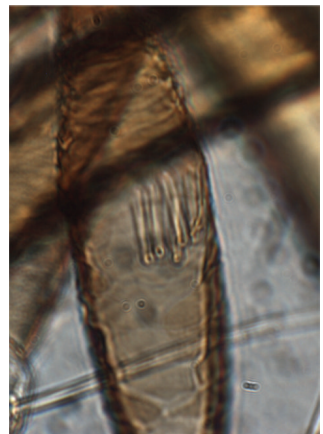

(e)

Figure 1: Pimachrysa fusca Adams, Joshua Tree National Park, Riverside Co., CA. (a, b, and c) Body, dorsal, illustrating the variation in the dorsal body markings among three third-instar specimens collected at the same locality on the same day. (d) Antenna (second instar), lateral, showing foreshortened flagellum typical of nothochrysine larvae. (e) Labial palps (second instar), lateral, showing row of five sensilla on terminal segment.

hooked, and the primary cephalic setae of the Pimachrysa larvae are slightly blunt and thus similar to those of Hypochrysa. Finally, the Pimachrysa head and body markings resemble those of $H$. elegans; that is, larvae of both species have dark brown, elongate, dorsal head markings, and dark brown, sublateral stripes on the thorax, and abdomen (most noticeable on the L3; see Figures 1(a), 1(c), 2(a), 2(b), and 6).

However, a number of distinguishing features differentiate the larvae of the two genera. First, other than the cephalic setae, all other setae on the Pimachrysa larvae, especially the abdominal submedian setae (SMS), are short and acutely tipped, whereas on Hypochrysa they are short and blunt or clavate. Second, the jaws of Hypochrysa are longer than those of the Pimachrysa (Hypochrysa: 1.7-1.8x head length; Pimachrysa 1.4-1.6x head length). Third, the white-to-tan body coloration of the Pimachrysa larvae differs markedly from the green-to-bright green coloration of both $H$. elegans and $H$. africana. In our opinion, the above differences offer significant supporting evidence for the separate identity of the two genera.

\section{Description of Pimachrysa fusca Adams}

\subsection{Second Instar}

4.1.1. Body (Figures 2(a)-2(c)). Length $\sim 5.5 \mathrm{~mm}$ (measured in lateral view through spiracles), height $\sim 0.94 \mathrm{~mm}$ (thickest section of abdomen). Cream-colored-to-white with dark brown dorsolateral and lateral markings on each segment; lateral surface with brown dorsal band above lateral band of white and lower narrow band of dark brown; pronotal sclerites brown. All setae short, smooth, pale, of two types: "simple" = with acute tip (most setae) or "slightly blunt" = with slightly rounded tip (some primary cranial setae). Thoracic, abdominal setae often with swollen, lightly sclerotized setal bases (Figures 3(a) and 3(c)), these setal bases becoming increasingly larger and more sclerotized posteriorly.

4.1.2. Head (Figures 2(a)-2(c), 4(a), and 4(b)). Dorsum white, with dark brown markings as in figures; frontal marking elongate, without lateral arms; mesal epicranial and/or postfrontal markings reduced to pair of posterior spots below frontal marking; lateral epicranial marking elongate, extending to lateral side of antennal base; eyes with stemmata clear, integument between stemmata, surrounding all stemmata dark brown to black; cranium roughly triangular, with posterior margin (dorsal) rounded, ventral margin elongate; width (across eyes) $\sim 0.79 \mathrm{~mm}$, length (dorsum) $\sim 0.64 \mathrm{~mm}$, depth (midregion to top of eye) $\sim 0.20 \mathrm{~mm}$; base mostly exposed. Clypeolabral area elongated distally, extending over basal segment of labial palps; with large brown mark mesally. All dorsal primary cephalic setae present except S10 and S12; S11 long, S3 short and shifted mesally, others of medium length, most slightly blunt apically; Vx setae obscured by cervix, probably 


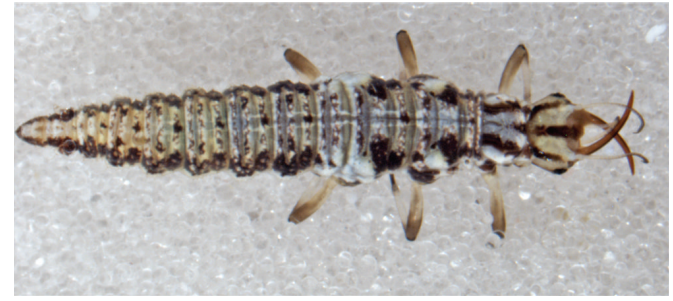

(a)

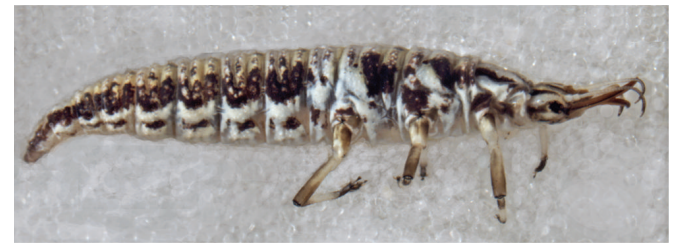

(b)

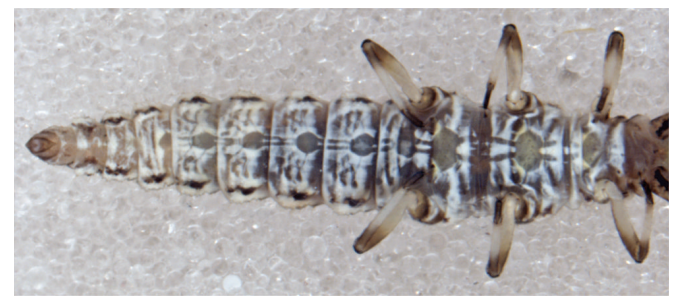

(c)

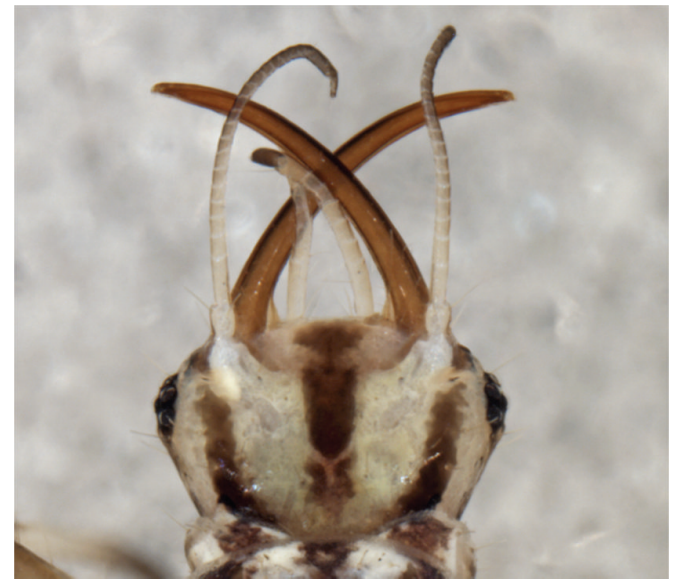

(d)

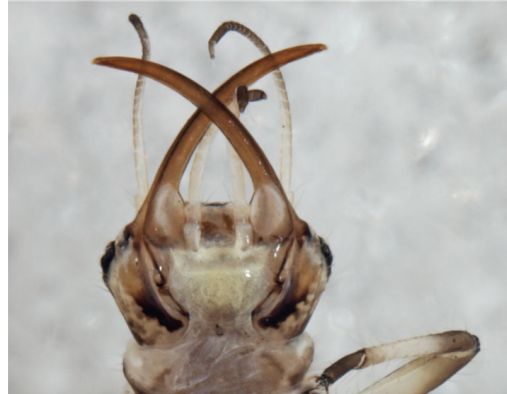

(e)

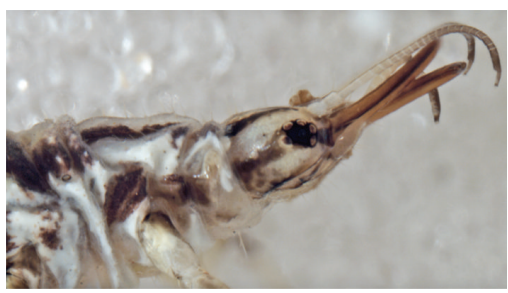

(f)

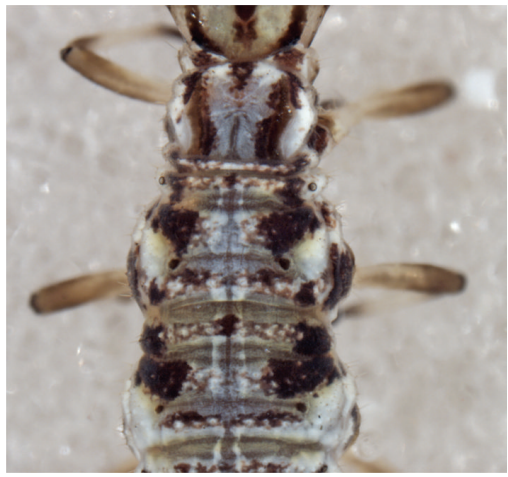

(g)

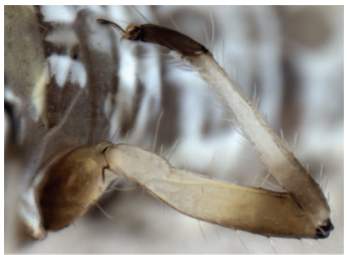

(h)

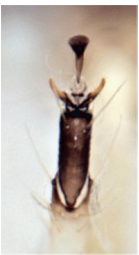

(i)

Figure 2: Pimachrysa fusca Adams, second instar (Joshua Tree National Park, Riverside Co., CA). (a) Body, dorsal. (b) Body, lateral. (c) Body, ventral. (d) Head, dorsal. (e) Head, ventral. (f) Head, lateral. (g) Thorax, dorsal. (h) Hind leg, anterior. (i) Tarsus, dorsal.

with three small setae (as drawn on Figure 4(a)) or two setae and pore; labrum with three pairs of setae (one mesally, two laterally); dorsum without secondary setae.

Cervix full laterally, ventrally, slightly withdrawn from cranium dorsally; with row of 6-8 pairs of setae along anterior margin, dorsum to venter.

4.1.3. Cephalic Appendages (Figures 2(d)-2(f), 4(a), and 4(b)). Mandible long, thin, length $\sim 0.88-0.90 \mathrm{~mm}$ (along curved exterior margin), length $0.74-0.78 \mathrm{~mm}$ (straight line), width (at base) $0.10-0.13 \mathrm{~mm}$; ratio mandible length (curved margin) to head width $=1.11-1.13$; ratio mandible length (curved margin) to head length (dorsal) $=1.37-1.40$. Mandible very slightly upturned distally, with single acute basolateral seta; terminus sharp, with four very small teeth. Antennal length $0.94 \mathrm{~mm}, \sim 1.5 \mathrm{x}$ length of cranium; width $\sim 0.04 \mathrm{~mm}$ (at widest part of pedicel); scape with relatively straight sides, two pairs of distal setae (one lateral, other dorsal); pedicel annulate, with 18-22 medium-length annulations mesally, one elongate annulation basally, one distally, distal annulations with two small setae, one lateral, one mesal, distomesal margin 


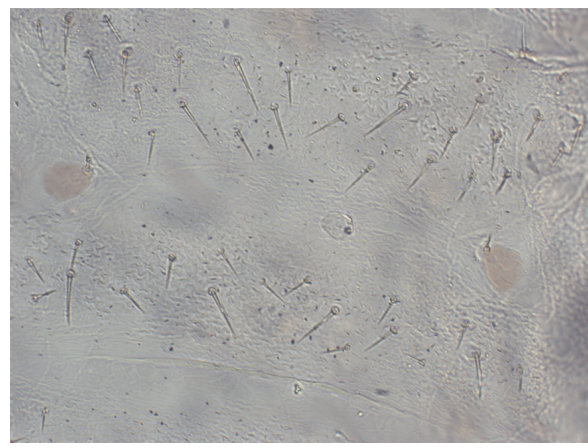

(a)

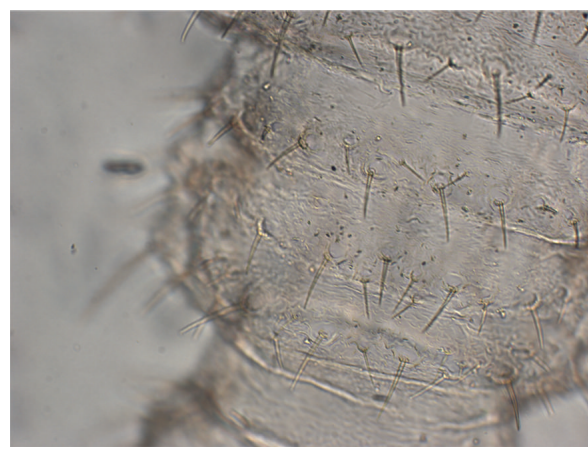

(c)

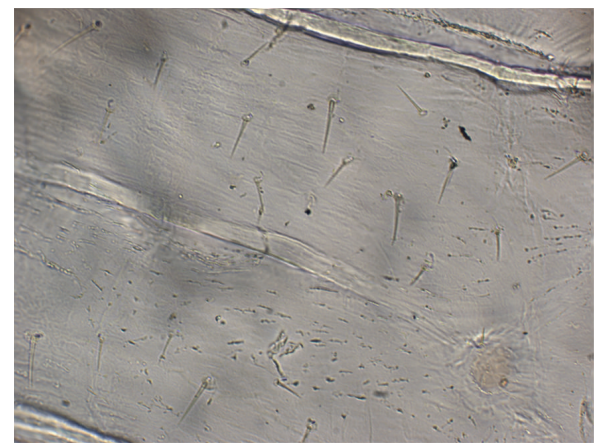

(b)

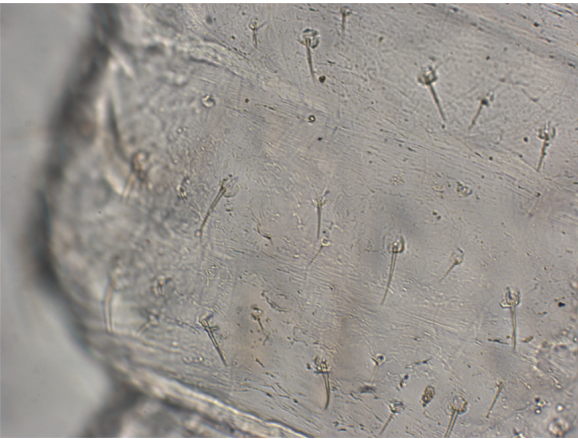

(d)

Figure 3: Pimachrysa fusca Adams (Joshua Tree National Park, Riverside Co., CA), thoracic (a, b) and abdominal (c, d) integument and setae, dorsal. Note slight differences in size of setae and chalazae between second $(a, c)$ and third $(b, d)$ instars. All images are the same magnification $(\times 100)$.

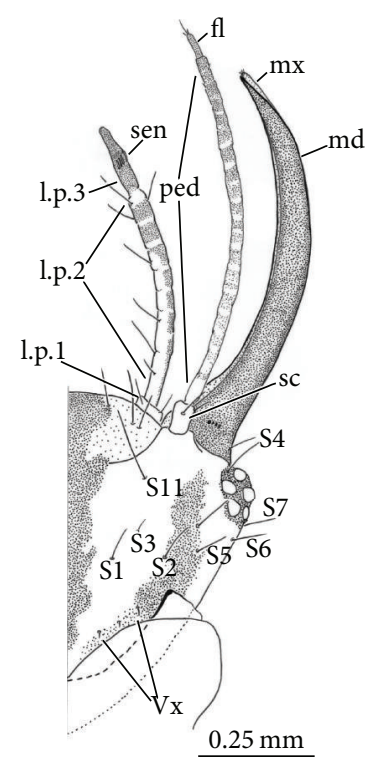

(a)

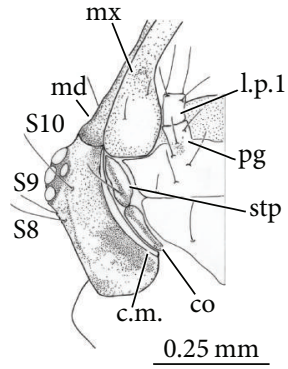

(b)

Figure 4: Head of Pimachrysa fusca Adams, second instar. (a) Dorsal. (b) Ventral. co: cardo; c.m.: cranial margin; fl: flagellum; l.p.x: labial palps, number of segment; md: mandible; mx: maxilla; ped: pedicel; pg: palpiger; sc: scape; stp: stipes; Sx: primary seta number; Vx: Vx setae. 


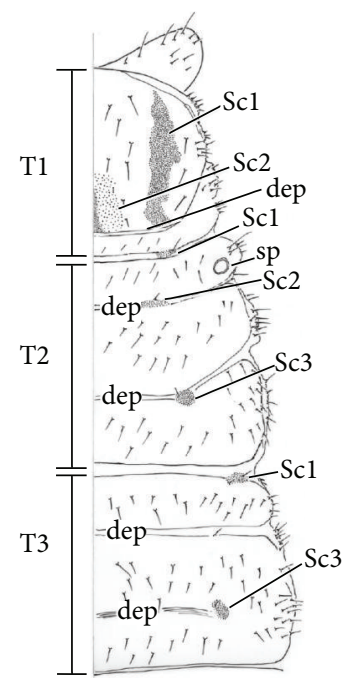

Figure 5: Thorax (dorsal) of Pimachrysa fusca Adams, second instar. dep: smooth-surfaced, intrasegmental depression between subsegments; Sc1, Sc2, and Sc3: first, second, and third primary sclerites of each segment; sp: spiracle; Tx: number of thoracic segment.

concave, bearing small claw; flagellum short, stubby, $\sim 0.08$ times length of pedicel, terminus with one short apical seta, surrounded by three or four microsetae. Labial palp long, slender, $\sim 0.85$ times length of mandible (straight line); basal segment with four distal setae, two dorsally, two ventrally; middle segment long, with long, undivided basal subsegment bearing three setae mesally, with $\sim$ six mesal subsegments, one mesal seta; terminal subsegment elongate, with two long setae distally, one basally; terminal labial segment $\sim$ one-third length of middle segment, slightly tapered distally, heavily sclerotized, no setae observed, ventrolateral margin with five closely spaced, elongate sensilla. Palpiger erect, with relatively straight sides, one mesal seta, one lateral seta; prementum broad, with three pairs of setae; cardo, stipes elongate, narrow, longitudinally arranged, cardo behind stipes, stipes with long seta.

4.1.4. Thorax (Figures 2(g) and 5). Lateral tubercles absent; dorsum with scattered, short, acute setae; pleurum with longitudinal, dorsal band of short bristles, spines; primary setae unidentified (except as noted below and in Figure 5). Legs (Figures 2(h) and 2(i)) white, with white-to-cream-colored setae; coxa dark brown; distal one-third of femur, basal one-fifth of tibia dark brown, with margins of all adjoining segments black distally; tarsus, claw, empodium dark brown.

Prothorax (T1) with two well-delineated subsegments separated by transverse depression. Paired Scl dark brown, elongate, extending full length of anterior subsegment, slightly narrow anteriorly, broader mesally, posteriorly, underlaid by dark brown, pigmented lateral stripe. Sc2 broadly ovate, extending from middle to posterior margin of anterior subsegment, with longitudinally elongate, brown marking posteromesally.
Mesothorax (T2) consisting of three well-delineated subsegments separated by distinct depressions. Anterior subsegment with pair of ovate sclerites (Scl) on anterior margin, with single lateral seta (probably S1Sc1); spiracles simple, sessile, circular, brown, with cylindrical atrium. Second subsegment with pair of small sclerites (Sc2) on anterior margin, each with single seta (S1Sc2), juxtaposed much smaller seta (S2Sc2); subsegment separated mesally from posterior subsegment by transverse depression with pair of large, brown, circular sclerites $(\mathrm{Sc}$ 3) laterally, each with small mesal seta (S1Sc3).

Metathorax (T3) consisting of three well-delineated subsegments separated by transverse depressions, each with pair of small setae laterally (probably S1Sc1, S1Sc2), but sclerites Sc1, Sc2 not identified. Sc3 medium-sized, brown, circular, with small mesal seta (S1Sc3).

4.1.5. Abdomen (Figures 2(a), 2(b), 2(c), and 6). Lateral tubercles (LTs), laterodorsal tubercles (LDTs) absent; spiracles (A1A8) circular, sessile, with simple atrium. Each segment (A1A7) roughly quadrangular, divided into three subsegments (or raised folds) separated by transverse depressions, anterior one extending to margin of segment; spiracle located laterally on dorsum of second subsegment; dorsum of all subsegments with acute submedian setae (SMS) on chalazae of small to medium sizes, arranged in transverse rows on each subsegment; pleura with patches of small, mostly acute, sometimes blunt setae, spines. A1: anterior subsegment short, dorsum with four pairs of SMS; second, third subsegments longer, similar in size to each other, each with row of $\sim$ ten to twelve pairs of SMS, most stemming from relatively small chalazae. A2-A7: dorsum of each subsegment with $\sim$ nine to twelve pairs of SMS; number and length of SMS, size (plumpness) of chalazae becoming progressively larger posteriorly. A7: first subsegment with four pairs of short SMS stemming from relatively large, plump chalazae; second and third subsegments each with five to seven pairs of SMS, most with plump chalazae. A8: segment divided into three subsegments; anterior subsegment narrow, well-delineated from subsequent subsegment; second, third subsegments not well-delineated from each other; dorsum of anterior subsegment with one pair of SMS, middle subsegment with two pairs of SMS; posterior subsegment with seven pairs of SMS; pleural region of segment with two, unsclerotized, lateral swellings, each bearing five medium length setae. A9: cylindrical, with two visible subsegments; anterior subsegment without setae anteriorly, with $\sim$ three pairs of robust dorsal setae posteriorly, lateral-most pair on swelling; posterior subsegment with row of $\sim$ seven pairs of medium length setae on robust chalazae posteriorly. A10: dorsum with $\sim$ six pairs of setae laterally, eight pairs of setae extending from posterior membrane on terminus. Venter with subsegmentation poorly demarcated; each segment (A1-A8) with two transverse bands of acute setae and spines (microsetae), pair of large, sublateral patches of relatively robust setae and spines (microsetae); setae often longer than those on dorsum, but without enlarged chalazae.

4.2. Third Instar. Similar to second instar except for measurements and noteworthy features, as follows. 


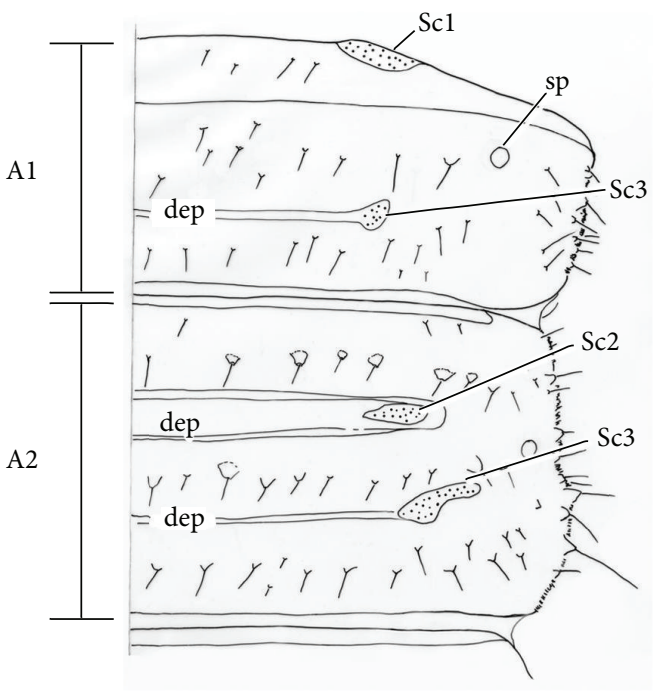

(a)

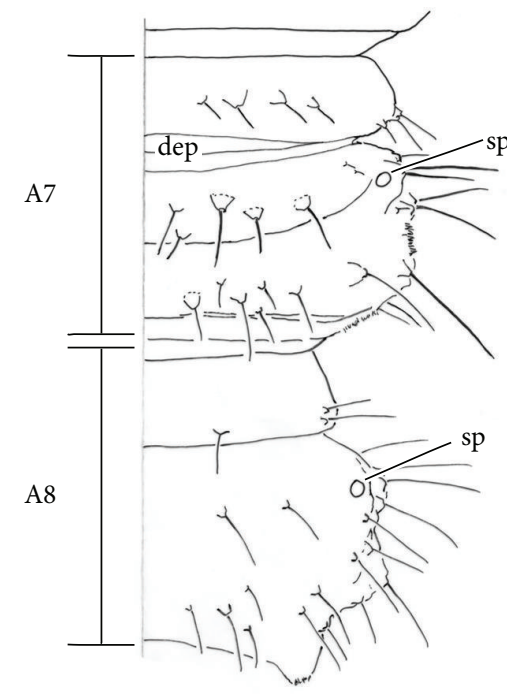

(c)

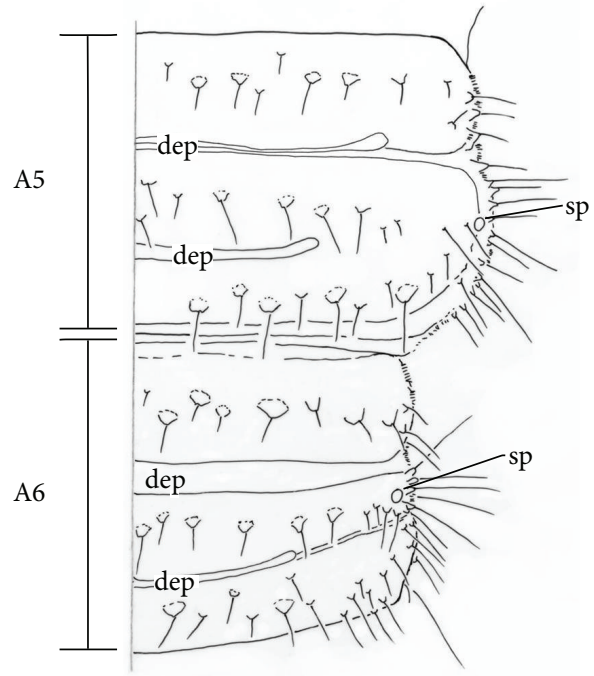

(b)

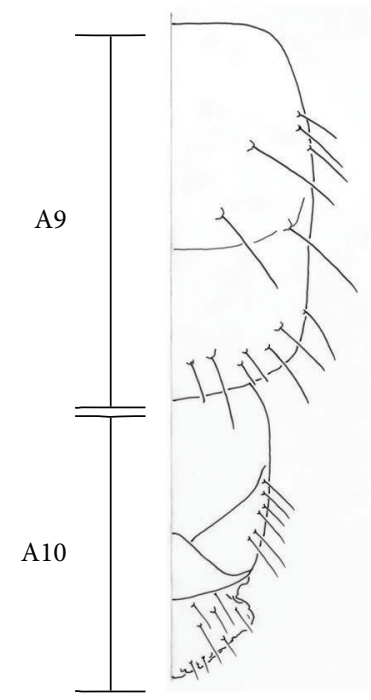

(d)

Figure 6: Abdomen (dorsal) of Pimachrysa fusca Adams, second instar. (a) First and second segments. (b) Fifth and sixth segments. (c) Seventh and eighth segments. (d) Ninth and tenth segments. Ax: number of abdominal segment; dep: smooth-surfaced, intrasegmental depression between subsegments; Scl, Sc2, and Sc3: first, second, and third primary sclerites of each segment; sp: spiracle.

\subsubsection{Body (Figures 1(a)-1(c), 7, 8(a), and 8(b))}

Body length (measured in lateral view through spiracles) $8.5-9.0 \mathrm{~mm}$ (L2: $5.5 \mathrm{~mm}$ ).

Body depth (thickest) 1.5-1.6 mm (L2: 0.94 mm).

Subsegmentation, setation, coloration as in L2.

Setal size approximately same as L2. Thoracic, abdominal setal bases sometimes slightly swollen, sclerotized (Figures 3(b) and 3(d)).

\subsubsection{Head (Figures $7(a)$ and $8(c)$ )}

Head width (across eyes) $0.77-0.78 \mathrm{~mm}$ (L2: $0.79 \mathrm{~mm})$.
Head length (dorsum) 0.63-0.64 mm (L2: 0.64 mm).

Head depth (midregion to top of eye) $0.18-0.19 \mathrm{~mm}$ (L2: $\sim 0.20 \mathrm{~mm}$ ).

\subsubsection{Cephalic Appendages (Figures 7(a) and 8(c))}

Mandible length (along curved exterior margin) 0.93-0.97 mm (L2: 0.88-0.90 mm).

Ratio, mandible length to head width $=1.19-1.29: 1$ (L2: 1.11-1.13:1).

Ratio mandible length to head length (dorsal) $=1.46-$ $1.57: 1$ (L2: 1.37-1.40).

Antennal length $\sim 0.96 \mathrm{~mm}, \sim 1.5 \mathrm{x}$ length of cranium (L2: $\sim 0.94 \mathrm{~mm}, \sim 1.5 \mathrm{x}$ length of cranium). 


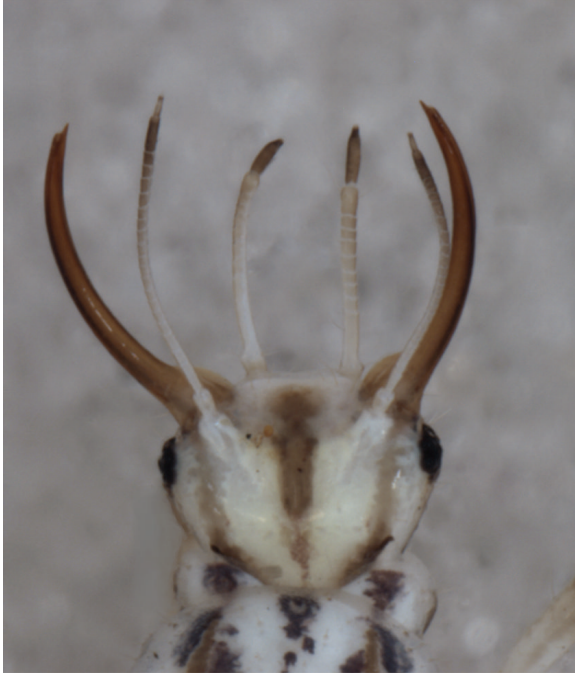

(a)

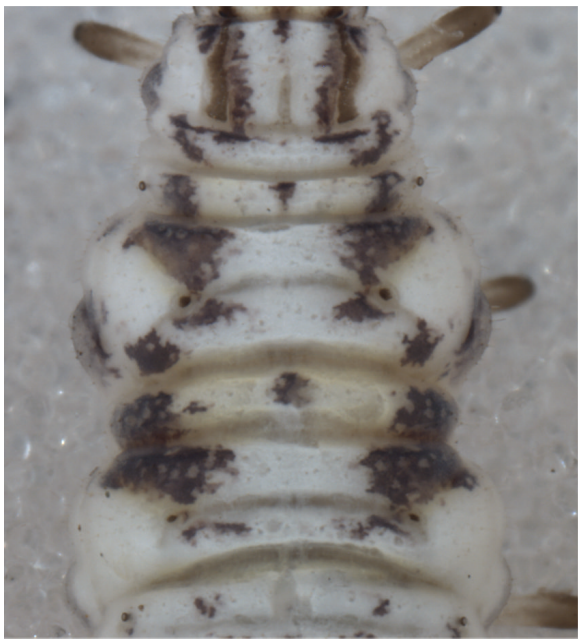

(b)

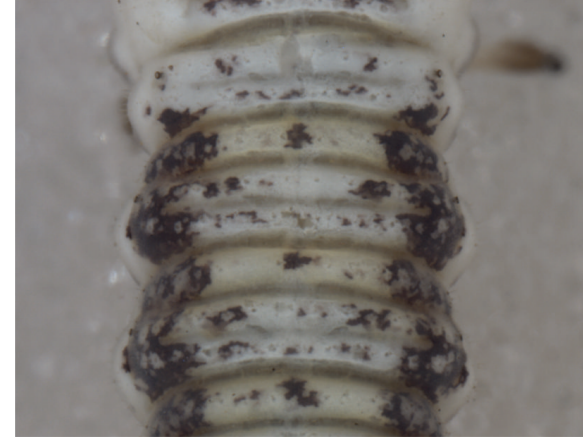

(c)

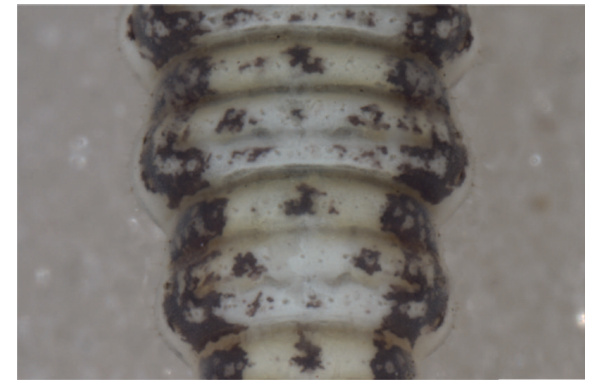

(d)

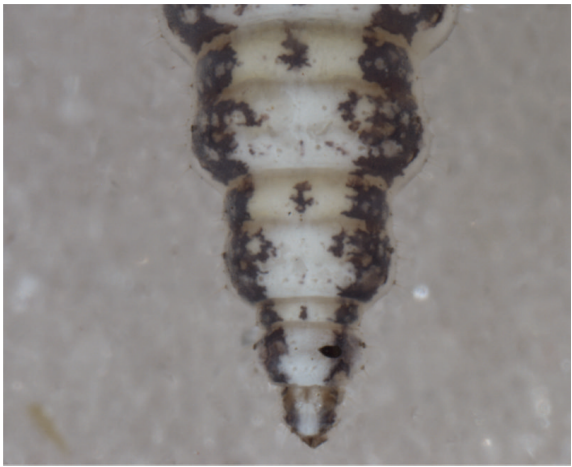

(e)

Figure 7: Pimachrysa fusca Adams, third instar (Joshua Tree National Park, Riverside Co., CA). (a) Head, dorsal. (b) Thorax, dorsal. (c) Abdominal segments 1 through 3, dorsal. (d) Abdominal segments 4 and 5, dorsal. (e) Abdominal segments 6 through 10, dorsal.

Flagellar length $0.06-0.07 \mathrm{~mm}$ (L2: $0.06 \mathrm{~mm})$; terminus with single very short apical seta, few, if any, surrounding microsetae.

Ratio, length of flagellum: length of pedicel $0.08: 1$ (L2: $0.07: 1$ ).

Ratio, length of labial palp:length of mandible (straight line) $\sim 0.78: 1$ (L2: $0.85: 1$ ).

Labial palp: middle segment with $\sim$ seven subsegments; ventrolateral margin of terminal segment with four to five closely spaced, elongate sensilla.

\subsubsection{Thorax (Figures $7(b)$ and 8 )}

Legs as in L2, except coxa white distally; basal onethird of tibia dark brown.
4.2.5. Abdomen (Figures 7(c)-7(e), 8(a), and $8(b)$ ). As in L2, with following exceptions:

A1: anterior subsegment dorsally with eight pairs of SMS; second, third subsegments each with row of $\sim$ twelve to fifteen pairs of SMS, most stemming from relatively small chalazae.

A2-A7: dorsum of each subsegment with nine to fifteen pairs of SMS-one to three more than L2.

A8: dorsum of anterior subsegment with two pairs of SMS; pleural region with single pair of broad, unsclerotized, lateral swellings, each bearing $\sim$ ten setae.

A10: basal portion of segment withdrawn and shorter than on $\mathrm{L} 2$.

Venter similar to L2. 


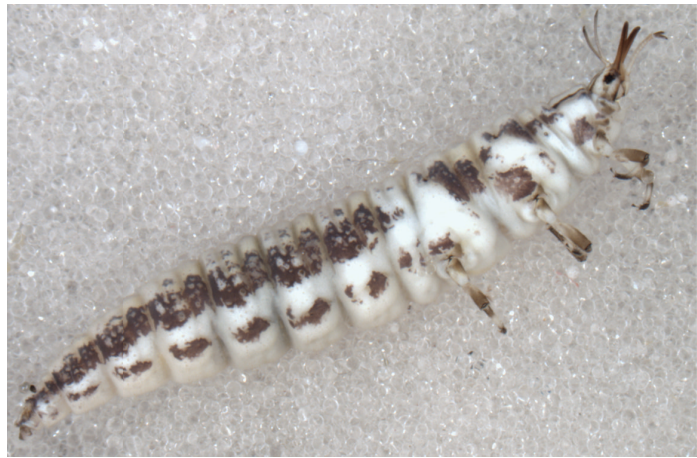

(a)

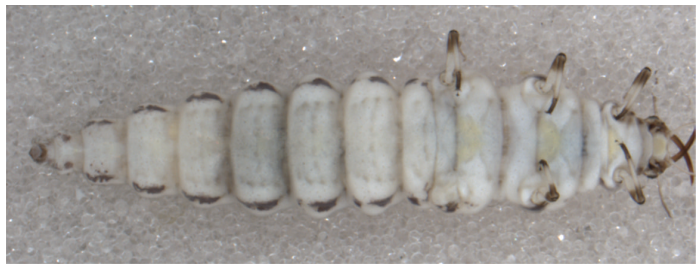

(b)

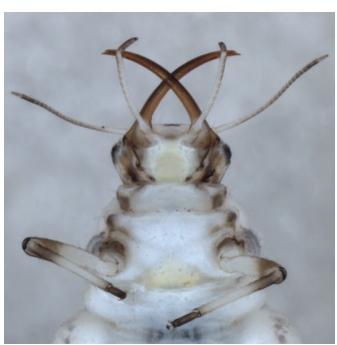

(c)

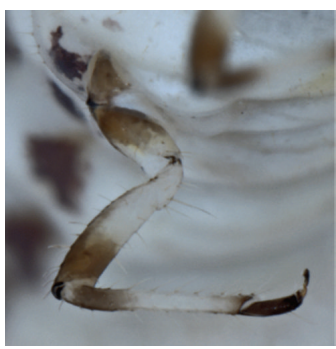

(d)

Figure 8: Pimachrysa fusca Adams, third instar (Joshua Tree National Park, Riverside Co., CA). (a) Body, lateral. (b) Body, ventral. (c) Head, prothorax, ventral. (d) Hind leg, anterior.

4.3. Size Differences between Second and Third Instars. Among our specimens, the L2 $(n=1)$ and L3 $(n=2)$ express some unusual variation in the relative sizes of body parts. The L3s, as expected, have much larger bodies than the L2, and yet the head size is almost identical among our specimens of the two instars. Also, while the L3 mandibles appear somewhat larger than those of the L2, the antennae and labial palps do not. In addition, the L3 setal size is approximately the same as in the L2, but the degree of enlargement and sclerotization of the abdominal setal bases is less than on L2 (Figures 3(a) and 3(c) versus 3(b) and 3(d)). Thus, the L3 setae seem less prominent than those of the L2 (Figure 3).

Given the small number of specimens, we are puzzled by the above variation in the relative sizes of larval body parts. First, we do not know whether our observations represent the normal situation for this species. Second, if it does, we suggest that the variation could be related to some unusual pattern of annual or seasonal variation in the availability of food or perhaps seasonal or generational differences in physical factors.
4.4. Cocoon (Figures 16(a) and 16(b)). White, oblong longitudinally, round transversely, with basal side spun directly against substrate; attached base with single layer of thin, transparent silk; upper surface with two layers of silk: inner layer: dense, relatively smooth and outer layer: sparse, mixed with debris.

\section{Comparative Information on Nothochrysa californica Banks}

Previously, the three instars and cocoon of N. californica were described in detail [16]. However, full comparisons with other described nothochrysine immatures are hampered because the descriptions lack adequate illustrations and several larval characters were not included. Here, we provide images and drawings of the $N$. californica second instar (Figures 9-13) and the following notes on all instars for use in comparisons. All observations and measurements below are based on one to two specimens per instar:

Mandible length (along curved exterior margin) $=$ L3: $1.63-1.66 \mathrm{~mm}$; L2: $1.15 \mathrm{~mm}$; L1: $0.80 \mathrm{~mm}$. 


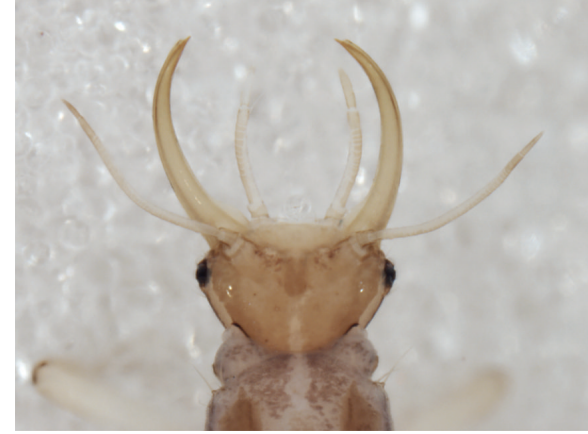

(a)

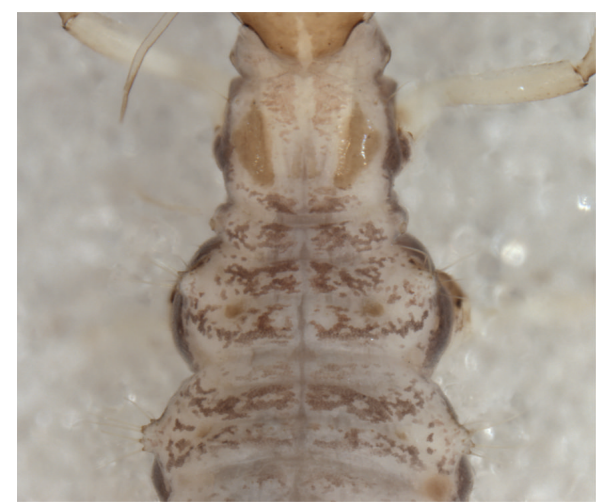

(c)

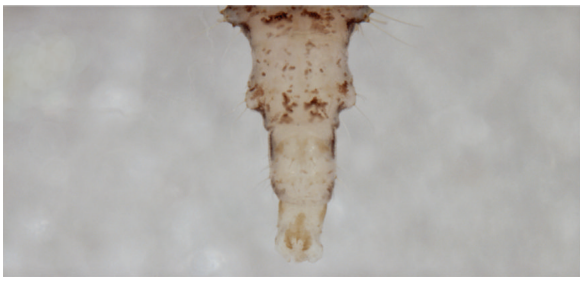

(f)

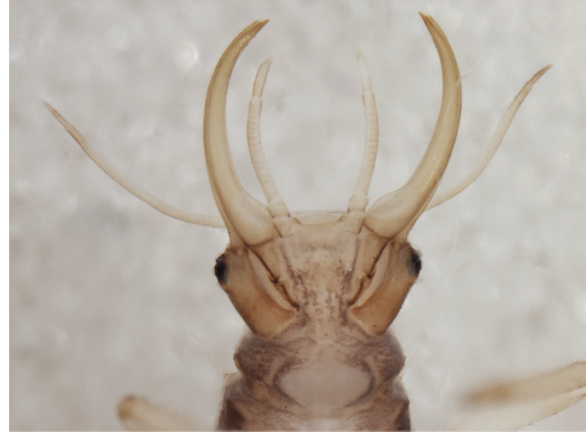

(b)

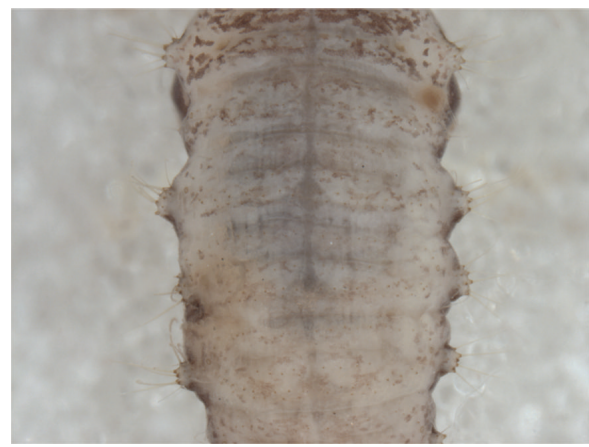

(d)

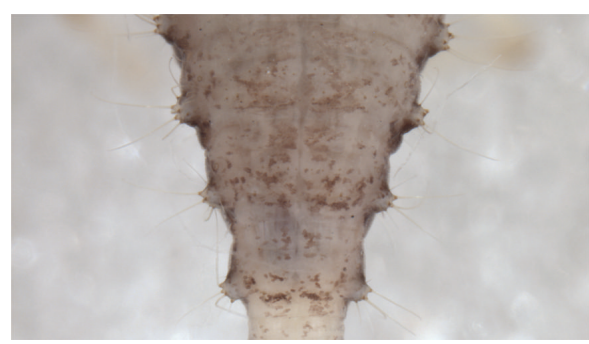

(e)

Figure 9: Nothochrysa californica Banks, second instar (Strawberry Canyon, University of California Campus, Alameda Co., CA). (a) Head, dorsal. (b) Head, ventral. (c) Thorax, dorsal. (d) Abdominal segments 1 through 4, dorsal. (e) Abdominal segments 5 through 7, dorsal. (f) Abdominal segments 8 through 10, dorsal.

Antennal length $=\mathrm{L} 3: \sim 1.65 \mathrm{~mm}, 1.5 \mathrm{x}$ length of cranium; L2: $1.24 \mathrm{~mm}, 1.8 \mathrm{x}$ length of cranium; L1: $0.84 \mathrm{~mm}, 1.6 \mathrm{x}$ length of cranium.

Pedicel with claw at tip = L3: yes; L2: yes; L1: yes.

Pedicel length = L3: $2.16 \mathrm{~mm} ;$ L2: $0.97 \mathrm{~mm} ; \mathrm{L} 1:$ $0.58 \mathrm{~mm}$.

Flagellar length $=$ L3: $0.18 \mathrm{~mm} ; \mathrm{L} 2: 0.17 \mathrm{~mm} ; \mathrm{L} 1:$ $0.17 \mathrm{~mm}$.

Length of flagellar seta $=\mathrm{L} 3: 0.03 \mathrm{~mm}$; $2: 0.03 \mathrm{~mm}$; L1: $0.04 \mathrm{~mm}$.

Labial palp, number of lateral sensilla on terminal segment = L3: 4; L2: 4-5; L1: 0 .

\section{Unique Larval Characters for Nothochrysinae}

Three larval features have been proposed as unique, and perhaps apomorphic, for the subfamily Nothochrysinae [5, 13, 18]. Given the recent data from Hypochrysa and now Pimachrysa, as well as our reexamination of the N. californica larvae, we were led to reevaluate the diagnostic and phylogenetic value of these characters. In addition, we propose an addition larval character that may be unique to the Nothochrysinae. For our larval comparisons, we used our own measured values and estimated others from published drawings (Dictyochrysa: Figure 3 by New [14]; N. capitata, N. fulviceps: Figures 46 and 47 by Monserrat and Díaz-Aranda [13]). 


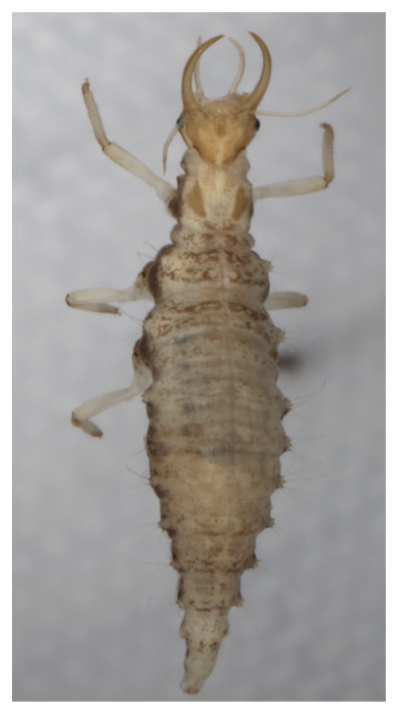

(a)

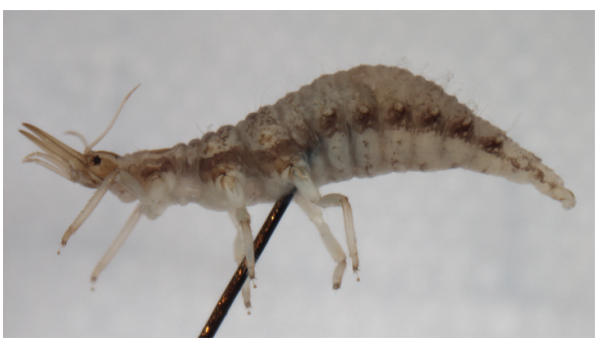

(b)

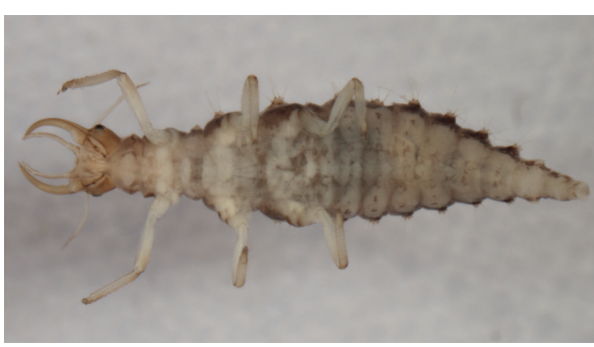

(c)

Figure 10: Nothochrysa californica Banks, second instar (Strawberry Canyon, University of California Campus, Alameda Co., CA). (a) Body, dorsal. (b) Body, lateral. (c) Body, ventral.

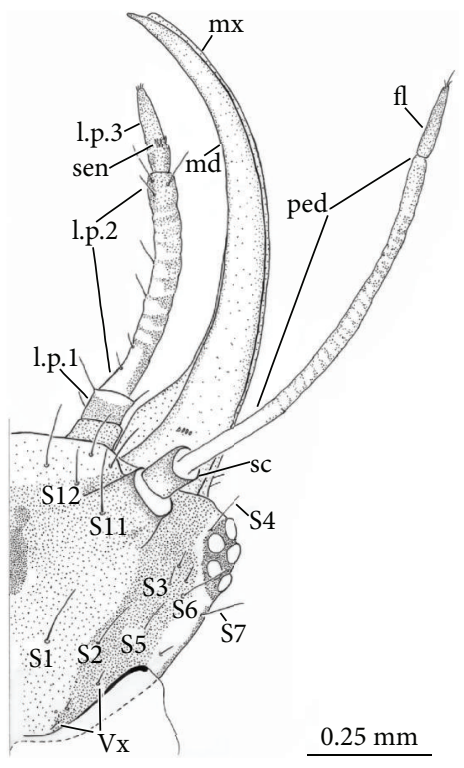

(a)

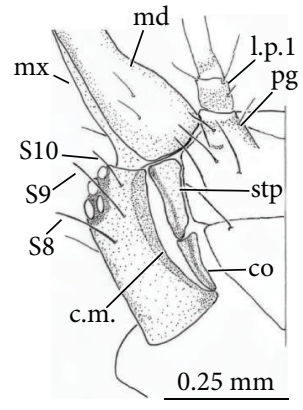

(b)

Figure 11: Head of Nothochrysa californica Banks, second instar. (a) Dorsal. (b) Ventral. co: cardo; c.m.: cranial margin; fl: flagellum; 1.p.1-3: labial palps, number of segment; md: mandible; mx: maxilla; ped: pedicel; pg: palpiger; sc: scape; sen: sensilla; stp: stipes; Sx: primary seta number; Vx: Vx setae. 


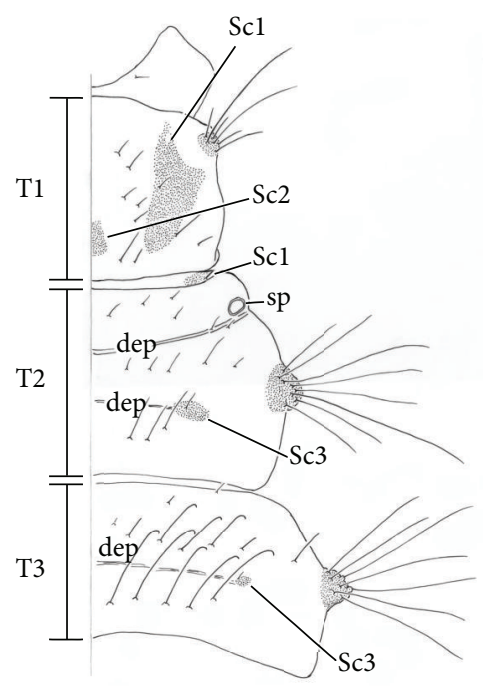

FIgURE 12: Thorax (dorsal) of Nothochrysa californica Banks, second instar. dep: smooth-surfaced, intrasegmental depression between subsegments; Sc1, Sc2, and Sc3: first, second, and third primary sclerites of each segment; sp: spiracle; Tx: number of thoracic segment.

6.1. Length of Flagellum. The nothochrysine antenna is characterized as having a terminal segment (flagellum) that typically is short and stubby, that is, approximately 10 or more times shorter than the pedicel; in larvae of Apochrysinae and Chrysopinae, the flagellum is considerably longer-only about 3-6 times shorter than the pedicel. For Hypochrysa and Pimachrysa second and third instars, as well as $N$. fulviceps and $N$. californica third instars, this character is consistent (their flagella are $\sim 12-20$ times shorter than the pedicel). Also, for the Dictyochrysa first instar, it is close at $\sim 9$ times shorter than the pedicel. However, there are interesting examples of interspecific and developmental variation in Nothochrysa. For example, the third instar of N. fulviceps, with a flagellum that is 12.5 times shorter than the pedicel, is consistent with the character, and the third instars of both N. capitata and N. californica (with flagella 8.3-9.1 times shorter than the pedicel) are near the 10x designation (Box 2). However, the flagella of the first and second instars of N. californica are noticeably longer, with the first instar being the most deviant (L3: flagellum 9.4-9.5 times shorter, L2: 5-7 times shorter, L1: only 4 times shorter than the pedicel; see Figure 14). Whether this pattern of developmental variation applies to the first instars of the European Nothochrysa species is unknown. However, if the old maxim "ontogeny recapitulates phylogeny" holds for this genus, the pattern could have phylogenetic importance.

6.2. Terminal Flagellar Setae. The nothochrysine flagellum is typified as bearing several, very small terminal setae; in contrast, the tip of the flagellum in Chrysopinae and Apochrysinae bears a single long seta with a robust base. Upon reevaluation, this trait holds for most of the known nothochrysine larvae-Dictyochrysa (L1, one species), Hypochrysa (L1, L2, and L3, one species each), Pimachrysa (L2, L3, one species,
Figure 14(a)), and Nothochrysa (L3, two European species). However, the structure differs in N. californica; in this species the flagellum terminates with a single elongate seta having a broad base (Figure 14(b)). The length of the terminal seta varies from 0.18 to 0.25 times the length of the flagellum. Again, the deviation from the typical nothochrysine pattern is most pronounced in the $N$. californica first instar. Unfortunately, the situation with the first and second instars of the European species is unknown.

6.3. Number of Sensilla on Terminal Segment of Labial Palp. Thus far, this character appears to be a consistent means of distinguishing nothochrysine larvae (second and third instars, Semaphoront B; there are no sensilla on the palps of the first instar). A number of four or more sensilla is typical of nothochrysine larvae. Three or fewer sensilla are present on larvae of Apochrysinae and Chrysopinae (note: this character is difficult to examine; it requires careful placement and often manipulation of a cleared specimen with labial palps in ventrolateral position and under sufficient lighting.)

6.4. Cocoon Structure. As described here, the Pimachrysa cocoon is sessile and strongly oblong; the basal membrane of the cocoon is single, thin, and highly transparent, whereas the upper layer is double, thick, and opaque. Among the Nothochrysinae, this cocoon structure is shared with Nothochrysa (Toschi 1965: N. californica); however, the situation is not clear for Hypochrysa. Hypochrysa africana shares the oblong ("barrel") shape of Pimachrysa and Nothochrysa (Duelli et al. 2010, as Kimochrysa), but it is unknown if its cocoon is sessile or entire. In any case, the Pimachrysa and Nothochrysa cocoons appear unlike the entire, more spherical cocoons spun by larvae of many Chrysopinae (Chrysopini, Leucochrysini) that we have observed.

6.5. Synapomorphies? Whether the above characters constitute apomorphies or symplesiomorphies is unconfirmed, and available evidence from the hemerobiids (the presumed sister group of Chrysopidae) is inconclusive. For example, among the hemerobiids, (1) the length of the flagellum exhibits intergeneric variability: in Boriomyia fidelis (Banks), the flagellum is short relative to the pedicel [19]; in Hemerobius species, it is elongate [20]; in Micromus species, it is indistinguishable from the pedicel [20]. (2) The length of the terminal flagellar seta also shows variability among hemerobiid genera: in $B$. fidelis and Drepanacra binocula (Newman) the flagellum is elongate [19, 21]; in Micromus it is short and accompanied by a very small seta [20]; in Hemerobius it is also short, but without a small seta [20]. To our knowledge, no hemerobiids have been shown to have a cluster of very short setae on the terminal antennal segment as found in Hypochrysa, Pimachrysa, and Dictyochrysa. (3) To our knowledge, lateral sensilla have not been reported from the terminal segment of hemerobiid labial palpi. They appear to be unique to Chrysopidae; however, the plesiomorphic number is unknown. (4) At this point, reports on the structural features of Neuropteran cocoons are not sufficient for meaningful phylogenetic comparisons. 


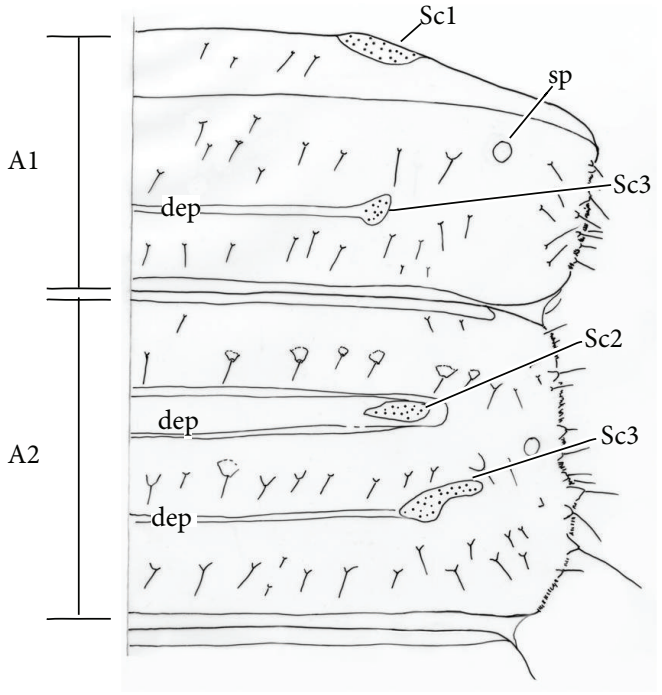

(a)

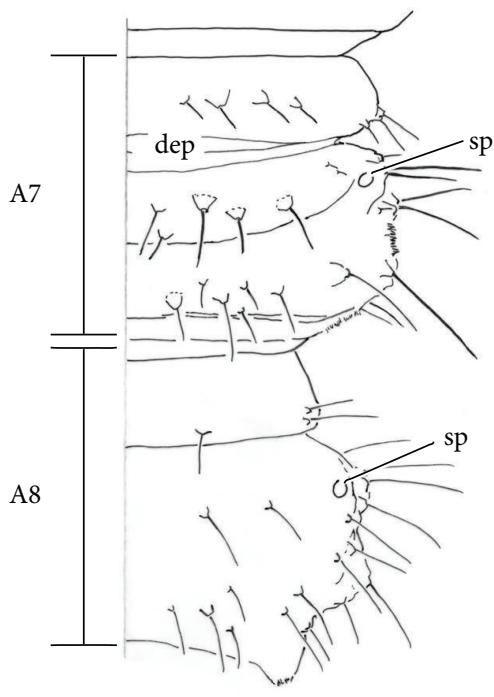

(c)

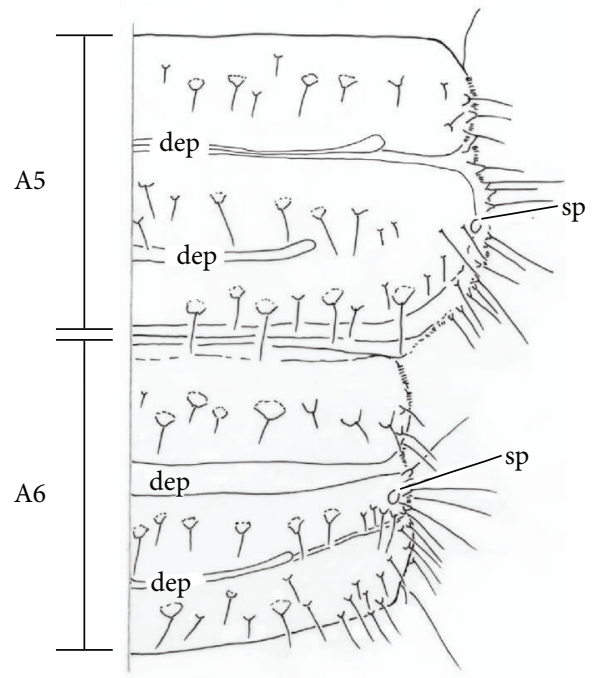

(b)

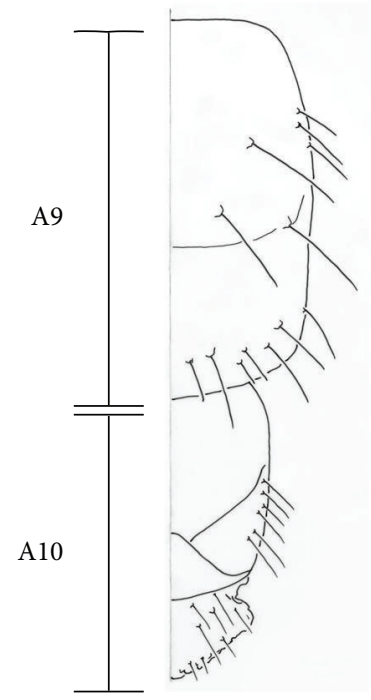

(d)

Figure 13: Abdomen (dorsal) of Nothochrysa californica Banks, second instar. (a) First and second segments. (b) Fifth and sixth segments. (c) Seventh and eighth segments. (d) Ninth and tenth segments. Ax: number of abdominal segment; dep: smooth-surfaced, intrasegmental depression between subsegments; Scl, Sc2, and Sc3: first, second, and third primary sclerites of each segment; sp: spiracle.

From the above, it is obvious that the choice of an out-group is of crucial importance for the interpretation of an apomorphic versus plesiomorphic state for the various larval characters. Unfortunately, there has been relatively little comparative systematic work that compares hemerobiid and chrysopid larvae (see [22]).

\section{Nothochrysine Phylogeny}

When Adams [1] revised the subfamily Nothochrysinae, it comprised seven extant genera and four fossil genera attributed to the Miocene. Later, two extant and eight fossil genera were added, and one extant genus was synonymized $[3,6,23]$. So, now the subfamily includes eight extant genera and twelve extinct fossil genera from the early Eocene (Ypresian) to the Miocene (Aquitanian, Burdigalian?).

Although Adams [1] included Nothochrysa within the subfamily because it retained many archaic and/or unusual features that are not expressed in the Chrysopinae, he pointed out that it has other advanced features that are more typical of Chrysopinae and Apochrysinae than Nothochrysinae (e.g., in its development of the pseudomedia and pseudocubitus, its wing folds). And recently, an extensive study of all known fossil chrysopids identified patterns of evolutionary progression in certain venational features among the nothochrysine fossils that support a distinct position for Nothochrysa [3]. But, the relationships among the extant genera remain unresolved.

Based on his morphological studies, Brooks [2] proposed that the extant genera of the subfamily fall into two groups. 


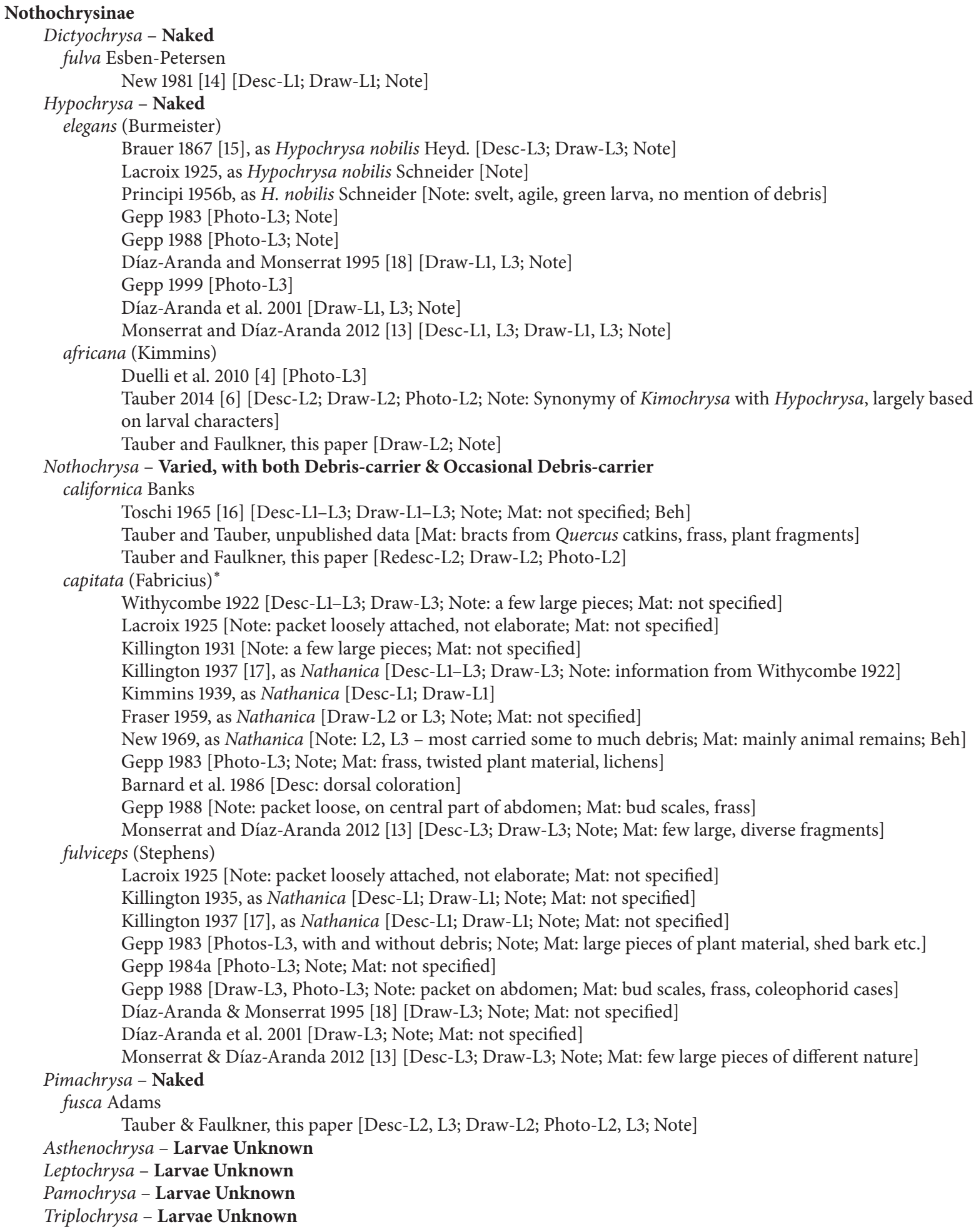

Box 1: Catalog of larval descriptions for Nothochrysinae (updated from Tauber et al. [5], where the citations not listed in the references here can be found). *See Monserrat and Díaz-Aranda 2012 [13] regarding problems in distinguishing N. capitata and N. fulviceps. 


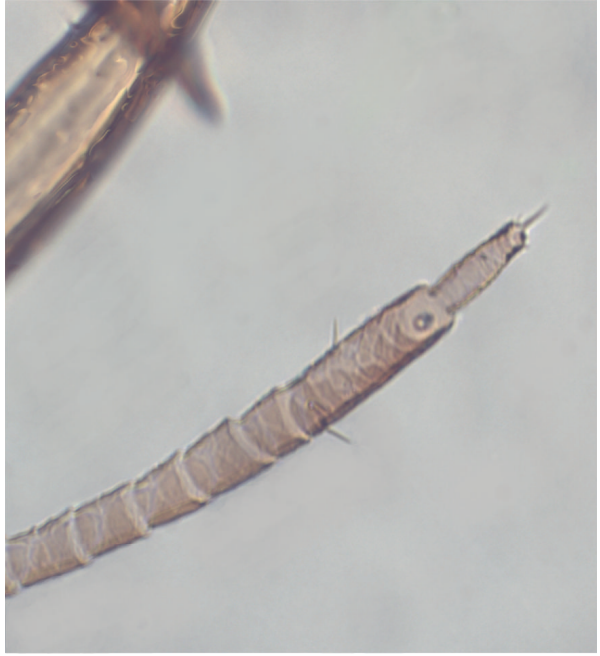

(a)

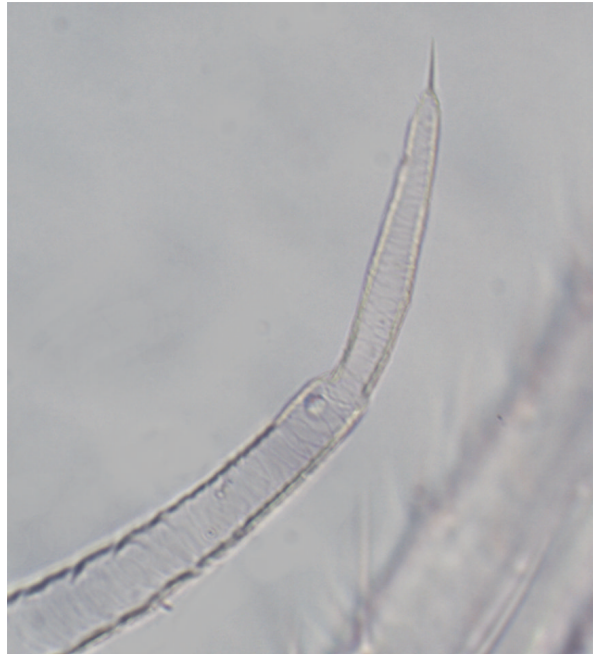

(b)

Figure 14: Flagellum and distal annulations of pedicel on (a) Pimachrysa fusca Adams and (b) Nothochrysa californica Banks, second instar antenna. Note: stubby flagellum and short terminal setae on $P$. fusca and elongate flagellum and single, more robust terminal seta on N. californica.

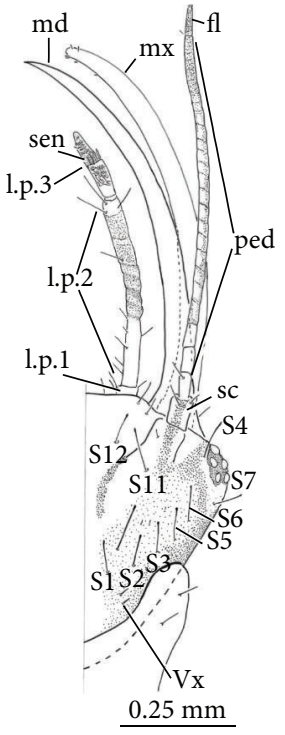

(a)

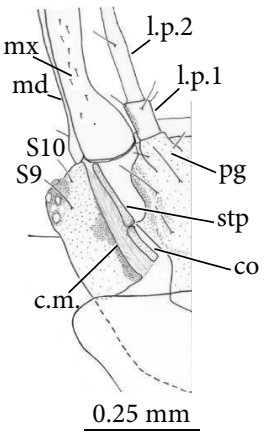

(b)

Figure 15: Head of Hypochrysa africana Kimmins, second instar. (a) Dorsal. (b) co: cardo; c.m.: cranial margin; fl: flagellum; l.p.x: labial palps, number of segment; md: mandible; mx: maxilla; ped: pedicel; pg: palpiger; sc: scape; sen: sensilla; stp: stipes; Sx: primary seta number; Vx: Vx setae.

In his scheme Nothochrysa was included with Dictochrysa and Hypochrysa, separate from Pimachrysa and Kimochrysa. These groupings subsequently were found to be weak; indeed Kimochrysa was synonymized with Hypochrysa [6]. Finally, in another study that used molecular and other data, Duelli et al. [4] proposed that the small bodied, pollen-feeding nothochrysine genera (e.g., Hypochrysa sen. lat. (including Kimochrysa), Pamochrysa, Pimachrysa, and Asthenochrysa) are very similar to each other. These authors concurred with the opinion that although Nothochrysa shows some affinities with these nothochrysine genera, they also resemble Apochrysinae and Chrysopinae in other respects. Given their findings, they conjectured that the small bodied, pollenfeeding nothochrysines represent the most ancient group of extant chrysopids. Moreover, they concluded that pollenfeeding is the likely ancestral condition for chrysopid adults.

It appears from the descriptions of now four of the eight nothochrysine genera (Box 1) that larval characters support the conclusions of Adams, Archibald and Makarkin, and Duelli et al. $[1,3,4]$. The pattern of larval differences between 
Pimachrysa (L2, L3)

Larvae naked, without a layer of camouflaging material.

Small to medium sized larvae (L2: $\sim 5.5 \mathrm{~mm}$; L3: 8.5-9.0 mm); shape fusiform, narrow, abdomen rounded, slender.

Cephalic markings obvious, lateral epicranial markings elongate; mesal epicranial and/or postfrontal markings reduced;

with a conspicuous median stripe.

Cephalic setae: all primary setae except S10 and S12 present; Vx obscure, probably two setae and a pore.

Antennae with terminal segment (flagellum) stubby, short (0.07-0.08x length of pedicel).

Mouthparts elongate (mandible $\sim 1.4-1.6 x$ longer than head capsule).

Mandible with single seta on basolateral margin.

Thorax without lateral tubercles.

Paired prothoracic sclerites (Sc1) elongate.

Metanotum with three distinct folds, each bearing a transverse band of unhooked setae with acute tips.

Legs fairly long in relation to body; tarsi dark brown throughout.

Abdominal segments II-VII without lateral tubercles, with some lateral setae slightly elongated, but without distinct lateral setae (LS).

Abdominal segments I-VII dorsally with transverse bands of numerous short, acute setae (SMS).

Abdominal segments V-VII without laterodorsal tubercles, without LDS (but with some laterodorsal setae slightly elongated).

Nothochrysa (all instars, unless noted otherwise)

Larvae usually bearing a camouflaging layer of debris.

Large larvae (L3: 9-12 mm; L2: 6-7 mm; L1: 4-5 mm); shape very slightly fusiform, abdomen convex, almost flattened to globular.

Cephalic markings obvious, epicranial markings large, fused with postfrontal markings, occupying almost the entire cephalic dorsal surface.

Cephalic setae: all primary setae including S12 present; $\mathrm{Vx}=$ two short anterior setae, pore, single posterior seta, in

longitudinally elongate row.

Antennae with terminal segment (flagellum) of variable length.

Pedicel length/flagellar length (= number of times flagellum shorter than pedicel):

N. capitata L3 $=\sim 8.8$ (from Figures $47-6[13]$ )

$N$. fulviceps L3 $=\sim 13.0$ (from Figures 47-12 [13])

N. californica $\mathrm{L} 1=\sim 3.6, \mathrm{~L} 2=5.1-6.2, \mathrm{~L} 3=9.4-9.5$

Flagellar terminus and terminal bristle variable.

N. capitata, N. fulviceps L3 with rounded flagellar terminus, very short terminal bristle (from Figure 1b [18], Figures 47-6, 47-12 [13])

$N$. californica L3-L1 with flagellum tapered, slightly elongate seta (setal length/flagellar length: L3 $=0.18, \mathrm{~L} 2=0.18$, $\mathrm{L} 1=0.25)$

Mouthparts noticeably longer than the head capsule.

N. capitata, N. fulviceps L3 (from Figures 46-1, 46-5 [13])

N. californica L1-L3 (mandible length/head length: L1 = 1.36, L2 $=\sim 1.60-1.65, \mathrm{~L} 3=1.30-1.48$ )

Mandible with two setae on basolateral margin.

Thorax with lateral tubercles present, carrying 1-2 or few elongate lateral setae (LS).

Paired prothoracic sclerites $(\mathrm{Scl})$ triangular.

Metanotum with transverse row of filiform (hooked or straight) setae posteriorly.

Legs very long in relation to body size; tarsi pale to dark brown.

Abdominal segments II-VII with lateral tubercles well developed, hemispherical, bearing filiform or hooked ("uncinate") setae (LS).

Abdominal segments VI-VII (sometimes also V, VIII) with laterodorsal tubercles present, bearing single long seta (LDS?)

(sometimes also a shorter seta).

Dorsum of abdominal segments I-VI with transverse rows or bands of hooked submedian setae (SMS).

Hypochrysa (L1, L3)

Larvae naked, without a layer of camouflaging material.

Small to medium sized larvae (L2: 6-7 mm); shape fusiform, narrow, abdomen rounded, slender.

Cephalic markings light or dark; lateral epicranial markings narrow, elongate; mesal epicranial and/or postfrontal markings faint, either with a conspicuous median stripe or with pair of small frontal markings.

Cephalic setae: all primary setae including S12 present; Vx obscure, probably two setae and a pore or three setae.

Antennae with terminal segment (flagellum) stubby, short ( 0.05-0.06x length of pedicel). 
Mouthparts elongate (mandible $1.7-1.8 x$ longer than head capsule).

Mandible with single seta on basolateral margin.

Labial palp with terminal segment bearing 4 lateral sensilla.

Thorax without lateral tubercles.

Paired prothoracic sclerites (Scl) elongate.

Metanotum with three distinct folds, each bearing a transverse band or row of unhooked, blunt to clavate (bacilliform) setae.

Legs of normal size in relation to body; tarsi dark brown thoughout.

Abdominal segments II-VII without lateral tubercles, without distinct lateral setae (LS).

Abdominal segments I-VII dorsally with transverse rows or bands of numerous short, blunt to clavate (bacilliform) setae

(SMS).

Abdominal segments V-VII without laterodorsal tubercles, without LDS.

Dictyochrysa (L1)

Larvae naked, without a layer of camouflaging material.

Small to medium sized larvae (L1: $\sim 2 \mathrm{~mm}$ ); shape fusiform, narrow, abdomen rounded, slender.

Cephalic markings unknown.

Cephalic setae: S12 absent; S8-10 unknown; Vx short.

Antennae with terminal segment (flagellum) rounded apically, pedicel length/flagellar length $=9$ (number of times flagellum

shorter than pedicel) (from Figure 3 [14])

Mouthparts nearly twice as long as head capsule.

Number of setae on mandible unknown.

Thorax without lateral tubercles.

Paired prothoracic sclerites (Sc1) triangular.

Metanotum with three distinct folds, each bearing a transverse band of unhooked setae with acute tips.

Legs fairly long in relation to body; tarsi dark brown throughout.

Abdominal segments II-VII without lateral tubercles.

Abdominal segments I-VII dorsally with transverse bands of blunt setae (SMS).

Abdominal segments V-VII without laterodorsal tubercles, without LDS.

Box 2: Summary of larval features of nothochrysine genera with described larvae. In addition to information in this paper; see [6, 13-18].

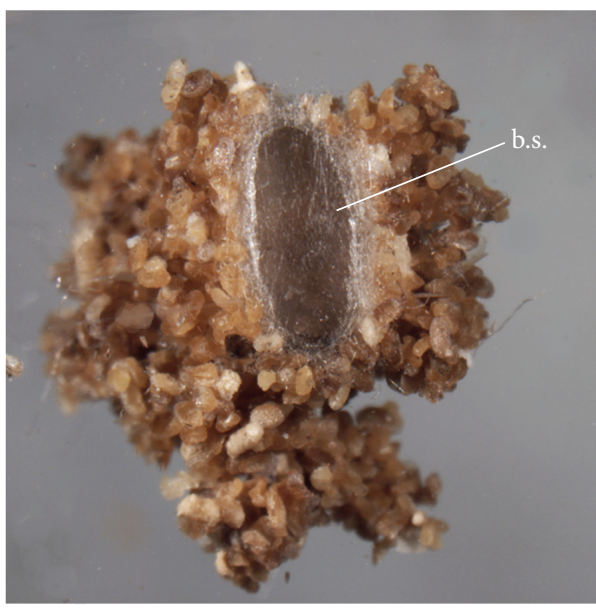

(a)

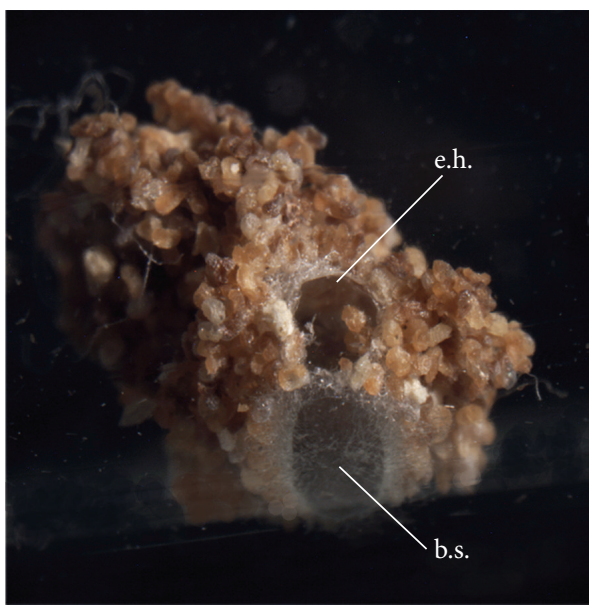

(b)

Figure 16: Pimachrysa fusca Adams, cocoon (Joshua Tree National Park, Riverside Co., CA). (a) Basal surface attached to glass vial. (b) Round exit hole on upper surface (head end) of cocoon. b.s.: basal surface; e.h.: exit hole.

Nothochrysa and the other known nothochrysine genera is consistent with the pattern of variation expressed in adult morphology. Most notable among the larval traits is the difference in larval life style between the small-bodied genera and Nothochrysa. Three of the small-bodied generaHypochrysa, Pimachrysa, and Dictyochyrsa-are now known to have naked larvae; they have narrow, fusiform bodies that lack lateral tubercles on the thorax and abdomen and are without laterodorsal tubercles on all abdominal segments. In marked contrast, the larvae of the European Nothochrysa species and the North American N. californica are debriscarriers; they have broad, somewhat flattened bodies, small 
but distinct, sclerotized, lateral tubercles on the thorax and abdominal segments A2 through A7, as well as other morphological adaptations for holding debris (Box 1).

It is noteworthy that within the family Chrysopidae, life style (larval nakedness versus debris-carrying) is associated with numerous morphological and behavioral adaptations, and yet it is an evolutionarily very labile trait. Debris carrying occurred very early in chrysopid evolutionary history [24], and both naked and debris-carrying forms have evolved numerous times within the family $[5,24]$. Thus, it could be argued that the characters above are redundant-all an expression of the naked versus debris-carrying life styles. However, it is important that, in addition to features associated with life style, the larvae of Nothochrysa species also differ from other nothochrysines in features that appear unrelated to their debris-carrying habit (Box 2). For example, as discussed above, larvae of Nothochrysa species show a broad range of interspecific and developmental variation in flagellar length and in the length and structure of the terminal seta(e) on the flagellum. They also differ in their head markings and in the shape and size of the pronotal sclerites. Each of these characters provides independent support that Nothochrysa is distinct from the smaller-bodied nothochrysine genera and that species within Nothochrysa present a series of intermediate conditions between the more archaic extant nothochrysines (e.g., Dictyochrysa, Hypochrysa, and Pimachrysa) and the Apochrysinae-Chrysopinae lineage(s). If this hypothesis proves true, not only would pollen-feeding likely be an ancestral adult feature of the extant Chrysopidae (as proposed by Duelli et al. [4]), but also nakedness would likely be their ancestral larval life style.

(Note: a previous description [6] of the H. africana second instar had some errors in the designation of setal numbers on the dorsum of the head. For accuracy in comparisons with Pimachrysa and other nothochrysine larvae, Figure 15 here corrects those errors.)

\section{Disclosure}

This study is part of Regional Project W-3185.

\section{Conflict of Interests}

The authors declare that there is no conflict of interests regarding the publication of this paper.

\section{Acknowledgments}

The authors thank Michael J. Tauber for his enthusiastic help collecting the second batch of larvae, Gilberto S. Albuquerque and Agatha J. Tauber for their comments on the paper, and Sheila and David Brodovsky as well as Jeanne Bellemin, Bob Allen, Michelle Rivers, and Marcus Hughes for logistical help and encouragement during the first field trip. The project benefitted from earlier funding by the National Science Foundation, the USDA Competitive Grants Program, the National Geographic Society, and Cornell University (to Maurice J. Tauber and CAT).

\section{References}

[1] P. A. Adams, "A review of the mesochrysinae and nothochrysinae (Neuroptera: Chrysopidae)," Bulletin of the Museum of Comparative Zoology, vol. 135, no. 4, pp. 215-238, 1967.

[2] S. J. Brooks, "An overview of the current status of Chrysopidae (Neuroptera) systematics," Deutsche Entomologische Zeitschrift, vol. 44, no. 2, pp. 267-275, 1997.

[3] S. B. Archibald and V. N. Makarkin, "A new species of Archaeochrysa Adams (Neuroptera: Chrysopidae) from the early Eocene of Driftwood Canyon, British Columbia, Canada," Canadian Entomologist, vol. 147, no. 4, pp. 359-369, 2015.

[4] P. Duelli, H. Hölzel, and M. W. Mansell, "Habitat and larvae of the enigmatic genus Kimochrysa Tjeder (Neuroptera: Chrysopidae) in South Africa," in Proceedings of the Tenth International Symposium on Neuropterology (22-25 June 2008, Piran, Slovenia), D. Devetak, S. Lipovsek, and A. E. Arnett, Eds., pp. 153-158, University of Maribor, Maribor, Slovenia, 2010.

[5] C. A. Tauber, M. J. Tauber, and G. S. Albuquerque, "Debriscarrying in larval Chrysopidae: unraveling its evolutionary history," Annals of the Entomological Society of America, vol. 107, no. 2, pp. 295-314, 2014.

[6] C. A. Tauber, "Nothochrysinae (Neuroptera: Chrysopidae): new larval description and generic synonymy, with a consideration of generic relationships," Psyche, vol. 2014, Article ID 839261, 10 pages, 2014.

[7] S. Winterton and S. de Freitas, "Molecular phylogeny of the green lacewings (Neuroptera: Chrysopidae)," Australian Journal of Entomology, vol. 45, no. 3, pp. 235-243, 2006.

[8] N. Haruyama, A. Mochizuki, P. Duelli, H. Naka, and M. Nomura, "Green lacewing phylogeny, based on three nuclear genes (Chrysopidae, Neuroptera)," Systematic Entomology, vol. 33 , no. 2, pp. 275-288, 2008.

[9] F. W. Stehr, "Techniques for collecting, rearing, preserving, and studying immature insects," in Immature Insects, F. W. Stehr, Ed., pp. 7-18, Kendall/Hunt Publishing Company, Dubuque, Iowa, USA, 1987.

[10] A. Rousset, Morphologie Céphalique des Larves de Planipennes (Insectes Névroptéroïdes), vol. 42 of Memoires du Museum National d'Histoire Naturelle Series A: Zoology, Éditions du Muséum, Paris, France, 1966.

[11] S. Tsukaguchi, Chrysopidae of Japan (Insecta, Neuroptera), S. Tsukaguchi, Nishinomiya-shi, Japan, 1995.

[12] C. A. Tauber, M. J. Tauber, and G. S. Albuquerque, "Berchmansus elegans (Neuroptera: Chrysopidae): larval and adult characteristics and new tribal affiliation," European Journal of Entomology, vol. 103, no. 1, pp. 221-231, 2006.

[13] V. J. Monserrat and L. M. Díaz-Aranda, "Los estadios larvarios de los Crisópidos ibéricos (Insecta, Neuroptera, Chrysopidae), nuevos elementos sobre la morfología larvaria aplicables a la sistemática de la familia," Graellsia, vol. 68, no. 1, pp. 31-158, 2012.

[14] T. R. New, "Some early stages of Dictyochrysa Esben-Petersen (Neuroptera, Chrysopidae)," Neuroptera International, vol. 1, no. 3, pp. 136-140, 1981.

[15] F. Brauer, "Larve von Hypochrysa nobilis Heyd," in Verhandlungen der Kaiserlich-Königlichen Zoologisch-Botanischen Gesellschaft in Wien, vol. 17, pp. 27-30, Zoologisch-Botanische Gesellschaft in Wien, 1867.

[16] C. A. Toschi, "The taxonomy, life histories, and mating behavior of the green lacewings of Strawberry Canyon (Neuroptera: Chrysopidae)," Hilgardia, vol. 36, no. 11, pp. 391-433, 1965. 
[17] F. J. Killington, A Monograph of the British Neuroptera, vol. 2, Ray Society, London, UK, 1937.

[18] L. M. Díaz-Aranda and V. J. Monserrat, "Aphidophagous predator diagnosis: key to genera of European chrysopid larvae (Neur.: Chrysopidae)," Entomophaga, vol. 40, no. 2, pp. 169-181, 1995.

[19] E. G. MacLeod, "The immature stages of Boriomyia fidelis (Banks) with taxonomic notes on the affinities of the genus Boriomyia (Neuroptera: Hemerobiidae)," Psyche, vol. 67, no. 12, pp. 26-40, 1960.

[20] A. H. Krakauer and C. A. Tauber, "Larvae of Micromus: generic characteristics and a description of Micromus subanticus (Neuroptera: Hemerobiidae)," Annals of the Entomological Society of America, vol. 89, no. 2, pp. 203-211, 1996.

[21] T. R. New, "The immature stages of Drepanacra binocula (Neuroptera: Hemerobiidae), with notes on the relationships of the genus," Australian Journal of Entomology, vol. 14, no. 3, pp. 247-250, 1975.

[22] J. D. Oswald and C. A. Tauber, "Recognition of larval Neuroptera. Preimaginal stages of the family Hemerobiidae," in Lacewings in the Crop Environment, P. K. McEwen, T. R. New, and A. E. Whittington, Eds., pp. 50-60, Cambridge University Press, Cambridge, UK, 2001.

[23] P. A. Adams and N. D. Penny, "Review of the South American genera of Nothochrysinae (Insecta: Neuroptera: Chrysopidae)," in Current Research in Neuropterology, Proceedings of the Fourth International Symposium on Neuropterology (24-27 June 1991, Bagnères-de-Luchon, Haute-Garonne, France), M. Canard, H. Aspöck, and M. W. Mansell, Eds., pp. 35-41, M. Canard, Toulouse, France, 1992.

[24] R. Pérez-de la Fuente, X. Delclòs, E. Peñalver et al., "Early evolution and ecology of camouflage in insects," Proceedings of the National Academy of Sciences of the United States of America, vol. 109, no. 52, pp. 21414-21419, 2012. 

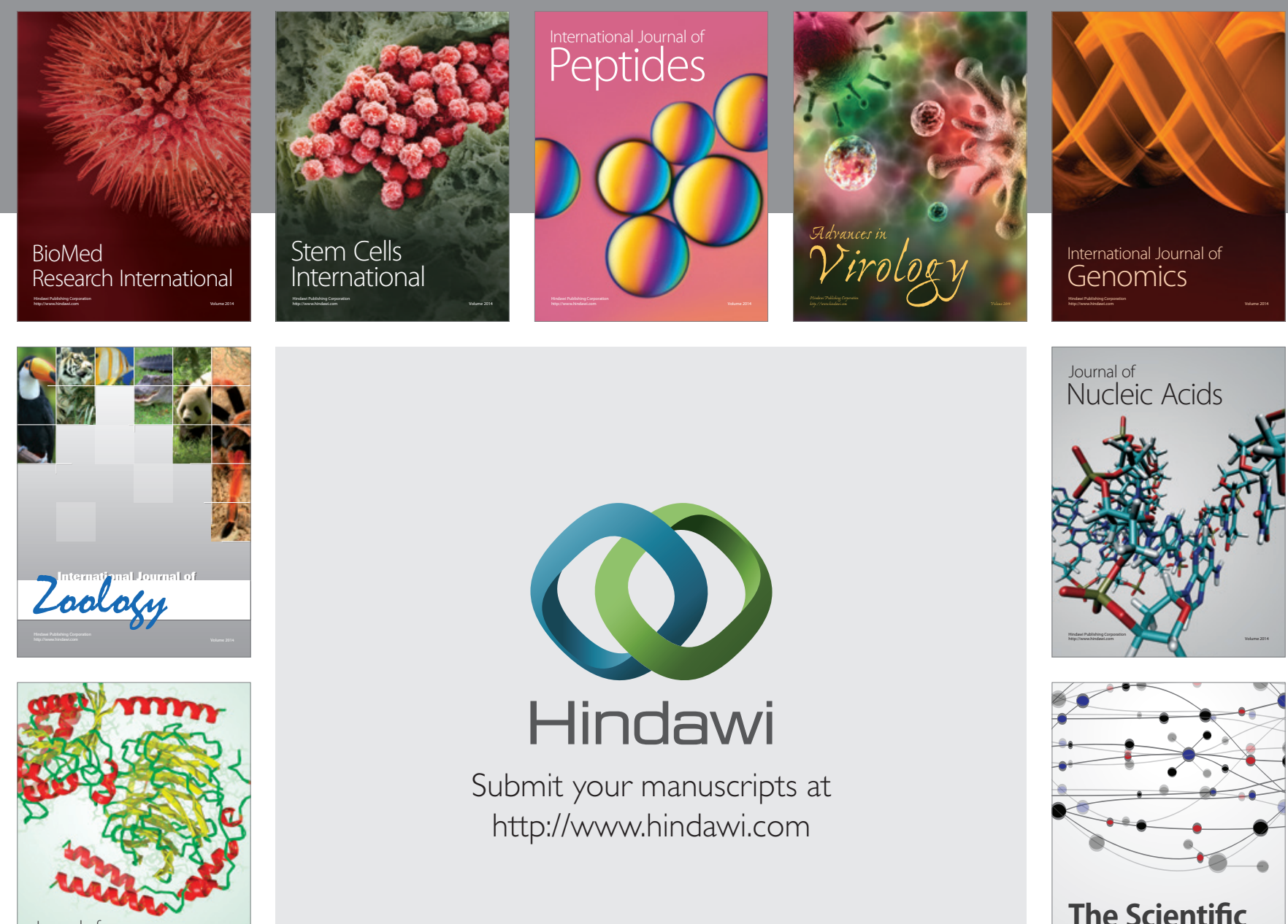

Submit your manuscripts at

http://www.hindawi.com

Journal of
Signal Transduction
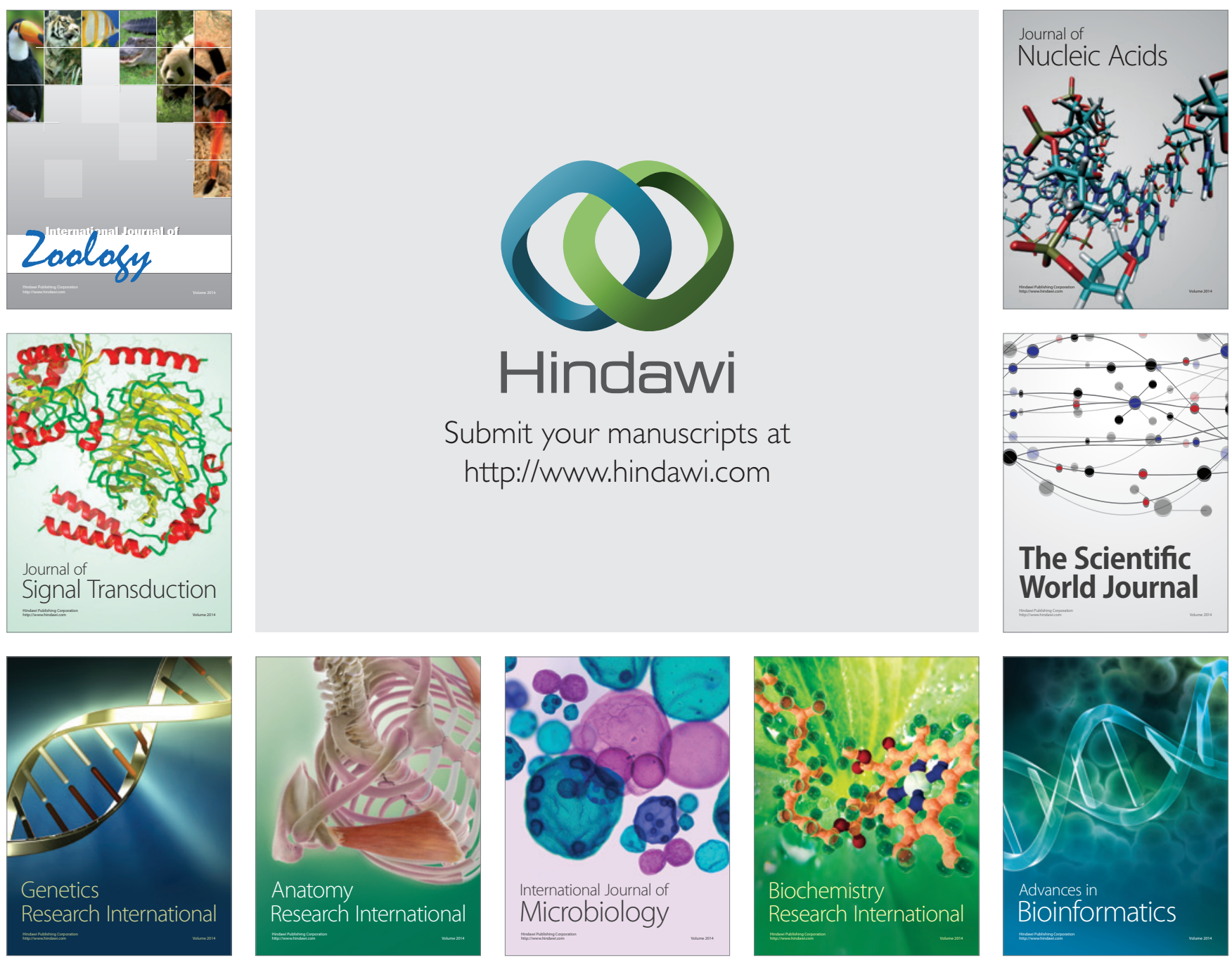

The Scientific World Journal
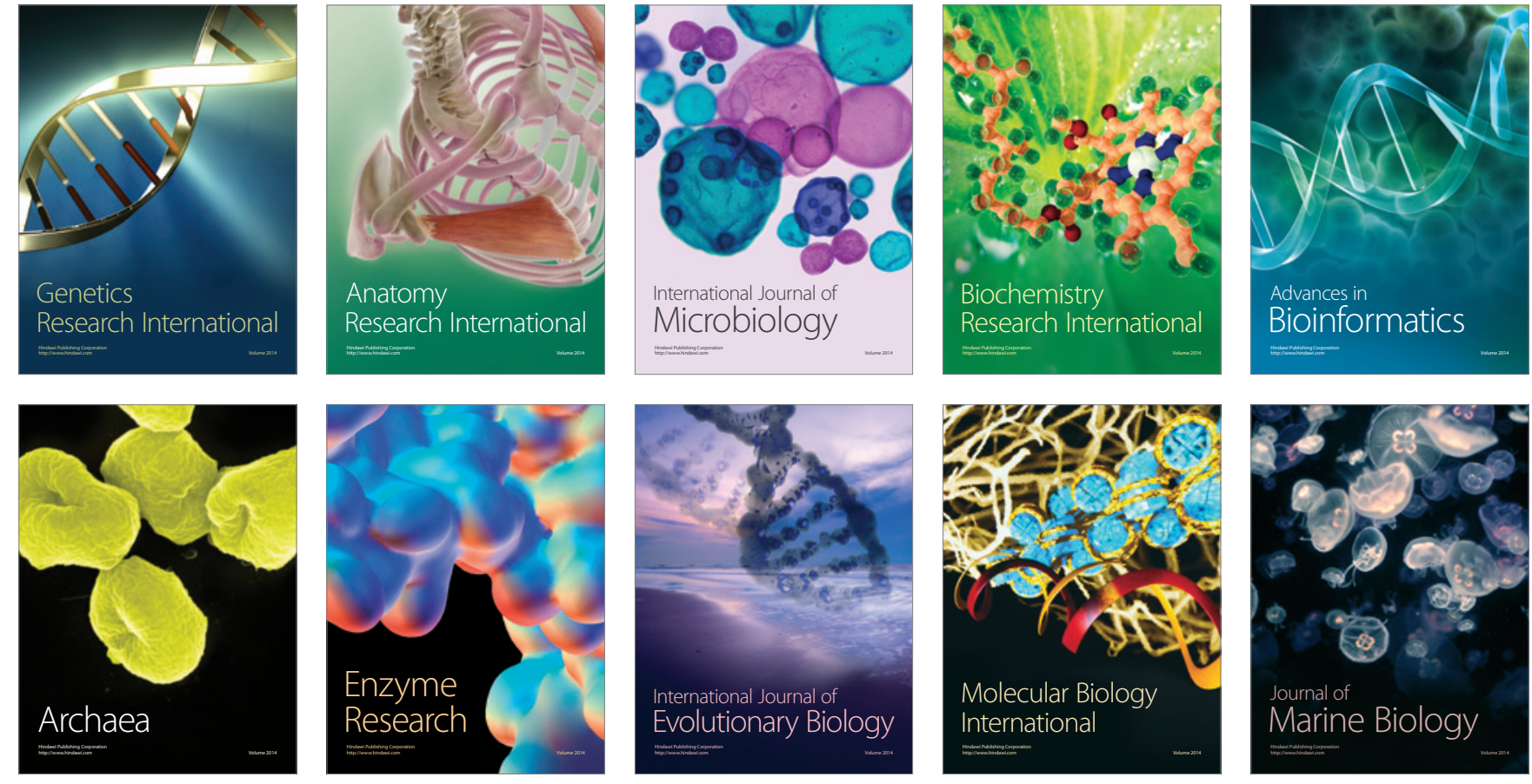